\title{
16. RADIOLARIANS FROM SITES 794, 795, 796, AND 797 (JAPAN SEA) ${ }^{1}$
}

\author{
Joanne M. Alexandrovich ${ }^{2}$
}

\begin{abstract}
Japan Sea ODP Leg 127 shipboard radiolarian biostratigraphic data are compiled and improved. The sequence of biostratigraphic events determined in sediments above the opal-A/opal-CT transition is illustrated graphically with depth-depth plots. The absence of biostratigraphic indicators from the North and subtropical Pacific and differences between the compositions of the Japan Sea and Pacific radiolarian assemblages suggest that the planktonic populations of the Japan Sea have been partially isolated from the Pacific since the late Miocene. Subtropical fauna in sediments younger than $\sim 1.8 \mathrm{Ma}$ at Site 797 record the occurrence of a paleo-Tsushima current. These same fauna record larger volumes of the paleo-Tsushima current, or warmer intervals during the colder glacial climate regime at Site 794. The variability of Pleistocene assemblage composition and preservation shows that radiolarian dissolution has played a large part in determining what is preserved. Preliminary taxonomic evaluations are made, and the stratigraphic and paleoceanographic implications of radiolarian species are discussed.
\end{abstract}

\section{INTRODUCTION}

Previous studies of Japan Sea radiolarian paleontology were limited to samples obtained with piston cores which span the last several hundreds to thousands of years (e.g., Morley et al., 1986; Oba et al., 1991), Miocene through Pleistocene sediments cored at four sites during Leg 31 of the Deep Sea Drilling Project (DSDP) (Ling, 1975), and Miocene and Pliocene deposits found on Japan (e.g., Nakaseko et al., 1965; Nakaseko, 1969). Piston cores from the Japan Sea contain stratigraphic sections that can be sampled at a rather high stratigraphic resolution, and Japan Sea Leg 31 cores contain relatively long records, but display sediment disturbances due to drilling problems (Karig, Ingle, et al., 1975). One of the principal objectives of Ocean Drilling Program (ODP) Japan Sea Leg 127 was to use the coring techniques of the advanced hydraulic piston corer (APC) and extended core barrel (XCB) to recover more complete stratigraphic sections that improve upon the recovery rates of DSDP Leg 31 sediments for paleontological and paleoceanographic study (Tamaki, Pisciotto, Allan, et al., 1990). Indeed, the sedimentary sections obtained during Leg 127, contain the longest, most continuous, records of radiolarian paleontology from the Japan Sea.

Radiolarians are found in sediment samples from Leg 127 sites that are as old as middle Miocene in age (Tamaki, Pisciotto, Allan, et al., 1990). The radiolarian assemblages in these samples display wide variability in both abundance and preservation. Younger (Pleistocene) assemblages show the highest variability in both preservation and abundance. Many Pleistocene samples are barren, and the dominant taxa in the assemblages from these samples change dramatically. The oldest radiolarians are found in sediments where opal-CT comprises the dominant siliceous phase and where diatoms are no longer preserved except in dolomitic nodules. Radiolarians are not found in sediments below the level of the diagenetic change from opal-CT to quartz. Radiolarians found in opal-CT sediments are not very abundant and rather poorly preserved, but were not completely obliterated from the opal-CT sediments because certain taxa dissolved relatively slowly and became coated and preserved by opal-CT precipitates. The details of radiolarian preservation within the opal-CT sediments from Japan Sea Leg 127 sites await further investigation.

\footnotetext{
${ }^{1}$ Pisciotto, K. A., Ingle, J. C.. Jr., von Breymann, M. T., Barron, J., et al., 1992. Proc. ODP, Sci. Results, 127/128, Pt. 1: College Station, TX (Ocean Drilling Program).

2 Department of Geological Sciences, Columbia University and Lamont-Doherty Geological Observatory, Palisades, NY 10964, U.S.A. (Present address: Department of Geology, Florida Atlantic University, Boca Raton, FL 33431, U.S.A.)
}

The intent of this study is to document radiolarian biostratigraphy and differences in assemblages among samples from the sediments of the Leg 127 Japan Sea sites in order to determine the sequence of radiolarian biostratigraphic events recorded in the cores and the paleoceanographic significance of assemblages with respect to the geologic history of the basin. Questions that concern how radiolarian assemblages reflect changes in sill depths, sources of nutrients, surface- and deep-water circulation, and the degree of isolation of the Japan Sea from the Pacific are addressed. Because distinctive preservational differences are observed among samples, these aspects of the radiolarian assemblages are examined in detail. Although there have been several thorough taxonomic studies of Japan Sea radiolarians, the taxonomy of Japan Sea radiolarians is still rather limited with respect to the diversity displayed by the Leg 127 assemblages. Therefore while summarizing the radiolarian paleontology and stratigraphy outlined in the Leg 127 shipboard reports (Tamaki, Pisciotto, Allan, et al., 1990), preliminary taxonomic evaluations are made on some dominant taxa and known stratigraphic indicators of Japan.

\section{SAMPLE PREPARATION AND DATA COLLECTION}

Samples were taken from Holes 794, 795, and 797 during Leg 127 for radiolarian paleontological analysis. The $10-\mathrm{cm}^{3}$ sample plugs taken from sediments above the opal-A/opal-CT transition were dried and weighed. They were subjected to treatment with $10 \%$ hydrogen peroxide, and if calcium carbonate material was present in the samples, with $\mathrm{HCl}$. Sodium pyrophosphate was added to the samples, which were then washed several times over a $63-\mu \mathrm{m}$ sieve. Residues of the sample-washing procedure were then settled onto glass slides using the standard technique of Moore (1973), and after drying under heat lamps, cover slips $(24 \times 50 \mathrm{~mm})$ were mounted to the slides using several drops of Canada Balsam. Slides were cured by heating on a hot plate. Although contamination of Leg 127 radiolarian samples by the frustules of large diatoms was obvious and troublesome during the slide-making procedure, samples were not treated with sodium carbonate or sodium hydroxide to break down and wash out the diatoms because this sample preparation step would probably alter the preservation of radiolarians in these samples as well.

Because of several complicating factors including limited time and the aggravating occurrence of Coscinodiscus marginatus in radiolarian slides made from samples stratigraphically below the opal dissolution transition zone (White and Alexandrovich, this volume), complete stratigraphic analyses that include total slide scans and tabulations of all important and identifiable radiolarian species were not performed for this study. Therefore, the stratigraphy presented here (Table 1) represents a 
summation of the stratigraphy presented in the Leg 127 Initial Reports (Tamaki, Pisciotto, Allan, et al., 1990), with some additional refinement of events. The refinement consists of a reduced sample-spacing uncertainty for several datum levels. This was accomplished by using Leg 127 shipboard radiolarian stratigraphy to identify the interval (core) where the stratigraphic event was found. Samples from that core were then scanned for the presence or absence of the indicator species, and the refined datum level is recorded in Table 1. Entire radiolarian slides from samples located stratigraphically above the opal dissolution transition zone (White and Alexandrovich, this volume) were scanned in order to observe preservational differences. Abundance and preservation in these samples are recorded (Tables 2, 3, and 4) following preservation and abundance guidelines outlined in the radiolarian section of the "Explanatory Notes" of Tamaki, Pisciotto, Allan, et al. (1990, p. 49). During slide scanning, the occurrence of distinctive features of the assemblages or specimens were observed. Samples from Hole 794A that contain warmwater assemblages are tabulated in Table 5. Radiolarian species are identified using previously established names and descriptions, and all radiolarian species discussed in this report are listed in the Appendix, with several important stratigraphic, preservation, and paleoceanographic indicators illustrated in the plates.

Because many opaline sponge spicules are larger than $63 \mu \mathrm{m}$, they are commonly observed in samples prepared for radiolarian paleontological analysis. Sponge spicules are quite abundant in samples prepared from Leg 127 sediments, and are also indicative of the degree of dissolution certain radiolarian assemblages have been sub- jected to (Alexandrovich and White, this volume). There is one type of opaline sponge spicule from Japan Sea Leg 127 sediments which is noteworthy in this report on radiolarians. Sterraster sponge spicules (Pl. 1, Figs. 1, 2, and 3) are quite common in Leg 127 Japan Sea sediments and in radiolarian samples. This type of sponge spicule was observed in radiolarian samples analyzed during Leg 127, but was largely ignored because it is not a radiolarian and represented a seemingly unimportant unknown. In several instances, however, this microfossil was found concentrated in sediments that were termed "bioclastic oozes" by the Leg 127 sedimentologists, but the bioclasts could not be positively identified during Leg 127 . The "bioclast" microfossil is positively identified here as a long-ranging (back to at least the Jurassic) sterraster sponge spicule belonging to the family Geodiidae, which has a broad latitudinal distribution and can be found in a variety of water depths (R. M. Finks, pers. comm., 1991). Because sterraster sponge spicules that are greater than $63 \mu \mathrm{m}$ are somewhat common in radiolarian samples, this sponge spicule has been erroneously identified and classified as a radiolarian by several workers (Spumellina incertae sedis Form A and B by Benson, 1966; Hataina ovata by Huang, 1967).

\section{RADIOLARIAN PRESERVATION}

Records of the preservation of Leg 127 Japan Sea radiolarian assemblages are very variable, reflecting several conditions of sediment deposition and sediment diagenesis. For this reason, the material

Table 1. Radiolarian biostratigraphy of Leg 127 sites by ODP sample designation and depth (m below seafloor). LO and FO refer to the last-occurrence and first-occurrence datum levels of the species, respectively. Depth interval is caused by sample spacing.

\begin{tabular}{|c|c|c|c|c|c|c|c|}
\hline & Hole 794A & Hole 794B & Hole 795A & Hole 795B & Hole 796A & Hole 796B & Hole 797B \\
\hline FO $L$ sp. cf. $L$ grande & $\begin{array}{l}\text { 1H-4. 60-62-1H-5, 44-57 } \\
5.1-6.55\end{array}$ & & $\begin{array}{l}2 \mathrm{H}-\mathrm{CC}-3 \mathrm{H}-\mathrm{CC} \\
18.8-28.3\end{array}$ & & $\begin{array}{l}1 \mathrm{H}-\mathrm{CC}-2 \mathrm{H}-\mathrm{CC} \\
3.2-12.7\end{array}$ & & $\begin{array}{l}3 \mathrm{H}-\mathrm{CC}-4 \mathrm{H}-1,118-120 \\
24.9-26.08\end{array}$ \\
\hline LO C. cabrilloensis & $\begin{array}{l}3 \mathrm{H}-6,60-62-3 \mathrm{H}-\mathrm{CC} \\
24.4-25.8\end{array}$ & & $\begin{array}{l}4 \mathrm{H}-\mathrm{CC}-5 \mathrm{H}-\mathrm{CC} \\
37.8-47.3\end{array}$ & & $\begin{array}{l}5 \mathrm{H}-\mathrm{CC}-6 \mathrm{H}-\mathrm{CC} \\
41.2-50.7\end{array}$ & $\begin{array}{l}\text { 5R-CC-6R-CC } \\
59.5-69.1\end{array}$ & $\begin{array}{l}4 \mathrm{H}-\mathrm{CC}-5 \mathrm{H}-\mathrm{CC} \\
34.4-43.9\end{array}$ \\
\hline LO $S$. mobusta & $\begin{array}{l}4 \mathrm{H}-6,59-62-4 \mathrm{H}-\mathrm{CC} \\
33.89-35.3\end{array}$ & & $\begin{array}{l}12 \mathrm{H}-4,70-72-12 \mathrm{H}-5,70-72 \\
109.5-111\end{array}$ & & $\begin{array}{l}10 X-C C-11 X-C C \\
78.3-88.1\end{array}$ & $\begin{array}{l}\text { 9R-CC-10R-CC } \\
97.9-252.7\end{array}$ & $\begin{array}{l}5 \mathrm{H}-\mathrm{CC}-6 \mathrm{H}-\mathrm{CC} \\
43.9-53.4\end{array}$ \\
\hline LO D. acquilonius & $\begin{array}{l}5 \mathrm{H}-4,59-61-5 \mathrm{H}-5,58-60 \\
40.39-41.88\end{array}$ & & $\begin{array}{l}10 \mathrm{H}-\mathrm{CC}-11 \mathrm{H}-\mathrm{CC} \\
94.8-104.3\end{array}$ & & $\begin{array}{l}8 \mathrm{H}-\mathrm{OC}-9 \mathrm{H}-\mathrm{CC} \\
58.7-68.4\end{array}$ & & $\begin{array}{l}8 \mathrm{H}-\mathrm{CC}-9 \mathrm{H}-3,118-120 \\
72.4-76.58\end{array}$ \\
\hline FO D. acquilonius & & & & & $\begin{array}{l}18 X-C C-19 X-C C \\
155.8-165.3\end{array}$ & & $\begin{array}{l}17 \mathrm{H}-\mathrm{CC}-18 \mathrm{H}-\mathrm{CC} \\
157.9-166.4\end{array}$ \\
\hline$L O L$ sp. aff. $T$, redondoensis & $\begin{array}{l}7 \mathrm{H}-\mathrm{CC}-8 \mathrm{H}-\mathrm{CC} \\
63.8-73.3\end{array}$ & & $\begin{array}{l}17 \mathrm{H}-\mathrm{CC}-18 \mathrm{H}-\mathrm{CC} \\
151.9-162.4\end{array}$ & & & & $\begin{array}{l}13 \mathrm{H}-\mathrm{CC}-14 \mathrm{H}-\mathrm{CC} \\
119.9-129.4\end{array}$ \\
\hline FO $T$. davisiana & $\begin{array}{l}8 \mathrm{H}-\mathrm{CC}-9 \mathrm{H}-\mathrm{CC} \\
73.3-82.8\end{array}$ & & $\begin{array}{l}16 \mathrm{H}-4,74-76-16 \mathrm{H}-\mathrm{CC} \\
147.54-151.8\end{array}$ & & $\begin{array}{l}10 X-C C-11 X-C C \\
78.3-88.1\end{array}$ & $\begin{array}{l}\text { 6R-CC-7R-CC } \\
69.1-78.8\end{array}$ & $\begin{array}{l}12-6,117-119-12 \mathrm{H} \cdot \mathrm{CC} \\
109.57-110.4\end{array}$ \\
\hline LO T. japonica & $\begin{array}{l}9 \mathrm{H}-3,59-61-9 \mathrm{H}-4,59-61 \\
76.89-78.39\end{array}$ & & $\begin{array}{l}11 \mathrm{H}-\mathrm{CC}-12 \mathrm{H}-\mathrm{CC} \\
104.3-113.8\end{array}$ & & $\begin{array}{l}9 \mathrm{X}-\mathrm{CC}-10 \mathrm{X}-\mathrm{CC} \\
68.4-78.3\end{array}$ & & $\begin{array}{l}11 \mathrm{H}-\mathrm{CC}-12 \mathrm{H}-\mathrm{CC} \\
100.9-110.4\end{array}$ \\
\hline FO S. langï̈ & $\begin{array}{l}10 \mathrm{H}-\mathrm{CC}-11 \mathrm{H}-\mathrm{CC} \\
92.3-101.8\end{array}$ & & $\begin{array}{l}19 \mathrm{H}-\mathrm{CC}-20 \mathrm{X}-\mathrm{CC} \\
171.9-181.6\end{array}$ & & $\begin{array}{l}12 X-C C-13 X-C C \\
97.7-117.2\end{array}$ & $\begin{array}{l}\text { 9R-CC-10R-CC } \\
97.9-252.7\end{array}$ & $\begin{array}{l}16 \mathrm{H}-\mathrm{CC}-17 \mathrm{H}-\mathrm{CC} \\
148.4-157.9\end{array}$ \\
\hline LO S. sp.cf. S. acquilonarium & $\begin{array}{l}10 \mathrm{H}-\mathrm{CC}-11 \mathrm{H}-\mathrm{CC} \\
92.3-101.8\end{array}$ & & $\begin{array}{l}15 \mathrm{H}-\mathrm{CC}-16 \mathrm{H}-\mathrm{CC} \\
142.3-151.8\end{array}$ & & $\begin{array}{l}10 X-C C-11 X-C C \\
78.3-88.1\end{array}$ & & $\begin{array}{l}16 \mathrm{H}-\mathrm{CC}-17 \mathrm{H}-\mathrm{CC} \\
148.4-157.9\end{array}$ \\
\hline LO T. akitaensis & & & $\begin{array}{l}16 \mathrm{H}-\mathrm{CC}-17 \mathrm{H}-\mathrm{CC} \\
151.8-151.9\end{array}$ & & & & $\begin{array}{l}11 \mathrm{H}-\mathrm{CC}-12 \mathrm{H}-\mathrm{CC} \\
100.9-110.4\end{array}$ \\
\hline FO T. akitaensis & & & $\begin{array}{l}30 X-C C-31 X-C C \\
279.1-288.8\end{array}$ & & & & $\begin{array}{l}23 X-C C-24 X-C C \\
214.3-224\end{array}$ \\
\hline FO $L$. sp. aff. $T$. redondoensis & $\begin{array}{l}15 \mathrm{H}-\mathrm{CC}-16 \mathrm{X}-\mathrm{CC} \\
139.8-149.5\end{array}$ & & $\begin{array}{l}34 \mathrm{X}-\mathrm{CC}-35 \mathrm{X}-\mathrm{CC} \\
317.6-327.2\end{array}$ & & & & $\begin{array}{l}22 \mathrm{X}-\mathrm{CC}-23 \mathrm{X}-\mathrm{CC} \\
204.8-214.3\end{array}$ \\
\hline LO B. bramlettei & $\begin{array}{l}10 \mathrm{H}-\mathrm{CC}-11 \mathrm{H}-\mathrm{CC} \\
92.3-101.8\end{array}$ & & & & & & \\
\hline LOS. peregrina & $\begin{array}{l}12 \mathrm{H}-\mathrm{CC}-13 \mathrm{H}-\mathrm{CC} \\
111.3-120.8\end{array}$ & & & & & & $\begin{array}{l}18 \mathrm{H}-\mathrm{CC}-19 \mathrm{H}-\mathrm{CC} \\
166.4-175.9\end{array}$ \\
\hline LO $T$. redondoensis & $\begin{array}{l}18 X-C C-19 X-1,60-62 \\
168.5-169.1\end{array}$ & & & & & & \\
\hline LO S. japonica & $\begin{array}{l}18 X-C C-19 X-1,60-62 \\
168.5-169.1\end{array}$ & & $\begin{array}{l}\text { 38X-CC-39X-CC } \\
356.2-365.9\end{array}$ & & & & $\begin{array}{l}\text { 20X-CC-21X-CC } \\
185.5-195.2\end{array}$ \\
\hline FO $T$. japonica & $\begin{array}{l}20 \mathrm{X}-\mathrm{CC}-21 \mathrm{X}-\mathrm{CC} \\
187.9-197.5\end{array}$ & & $\begin{array}{l}35 X-C C-36 \mathrm{X}-\mathrm{CC} \\
327.2-336.9\end{array}$ & & & & $\begin{array}{l}25 X-C C-26 X-C C \\
233.7-243.4\end{array}$ \\
\hline LO L nipponicum & $\begin{array}{l}20 X-C C-21 X-C C \\
187.9-197.538\end{array}$ & & & $\begin{array}{l}2 \mathrm{R}-\mathrm{CC}-3 \mathrm{R}-\mathrm{CC} \\
384.7-394.4\end{array}$ & & & $\begin{array}{l}24 X-C C-25 X-C C \\
224-233.7\end{array}$ \\
\hline LOS. delmontensis & $\begin{array}{l}21 X-C C-22 X-C C \\
197.5-207.2\end{array}$ & & & & & & \\
\hline LO T. anthopora & $\begin{array}{l}25 X-C C-26 X-C C \\
236.2-246\end{array}$ & & & $\begin{array}{l}7 \mathrm{R}-\mathrm{CC}-8 \mathrm{R}-\mathrm{CC} \\
432.8-442.4\end{array}$ & & & \\
\hline LO T. mammillaris & $\begin{array}{l}26 \mathrm{X}-\mathrm{CC}-27 \mathrm{X}-\mathrm{CC} \\
246-255.7\end{array}$ & & & $\begin{array}{l}7 \mathrm{R}-\mathrm{CC}-8 \mathrm{R}-\mathrm{CC} \\
432.8-442.4\end{array}$ & & & \\
\hline LO S, wolfii & $\begin{array}{l}27 X-C C-28 X-C C \\
255.7-265.4\end{array}$ & & & & & & \\
\hline LO L tochigiensis & $\begin{array}{l}28 X-1,60-62-28 X-2,60-62 \\
256.3-257.8\end{array}$ & & & $\begin{array}{l}7 R-C C-8 R-C C \\
432.8-442.4\end{array}$ & & & \\
\hline LO C. tetrapera & & $\begin{array}{l}\text { 5R-CC-6R-CC } \\
338.3-347.8\end{array}$ & & & & & \\
\hline
\end{tabular}


Table 2. Late Pliocene and Pleistocene age Hole 794A radiolarian samples with estimates of radiolarian abundance and preservation.

\begin{tabular}{|c|c|c|c|c|c|c|}
\hline \multirow{2}{*}{$\begin{array}{l}\text { Core, section, } \\
\text { interval }(\mathrm{cm})\end{array}$} & \multirow{2}{*}{$\begin{array}{l}\text { Depth } \\
\text { (mbsf) }\end{array}$} & \multirow{2}{*}{$\begin{array}{l}\text { Age } \\
\text { (Ma) }\end{array}$} & \multirow{2}{*}{$\begin{array}{c}\text { Slide weight } \\
(\mathrm{g})\end{array}$} & \multicolumn{2}{|c|}{ Radiolarians } & \multirow[b]{2}{*}{ Comments $^{\mathrm{a}}$} \\
\hline & & & & Abundance & Preservation & \\
\hline \multicolumn{7}{|l|}{$127-794 \mathrm{~A}-$} \\
\hline $1 \mathrm{H}-1,53-55$ & 0.53 & 0.015 & 6.00 & & & \\
\hline $1 \mathrm{H}-2,60-62$ & 2.10 & 0.060 & 5.49 & & & \\
\hline $1 \mathrm{H}-3,60-62$ & 3.60 & 0.103 & 4.19 & Barren & & \\
\hline $1 \mathrm{H}-4,60-62$ & 5.10 & 0.146 & 8.18 & Rare & Poor & High IR \\
\hline $1 \mathrm{H}-5,55-57$ & 6.55 & 0.188 & 10.44 & Rare & Moderate & $*$ \\
\hline IH-CC & 6.80 & 0.195 & & Few & Good & \\
\hline $2 \mathrm{H}-1,60-62$ & 7.40 & 0.212 & 10.16 & Barren & & * \\
\hline $2 \mathrm{H}-2,60-62$ & 8.90 & 0.255 & 5.59 & Few & Moderate & $*$ \\
\hline $2 \mathrm{H}-3,60-62$ & 10.40 & 0.298 & 9.93 & Rare & Poor & High IR \\
\hline $2 \mathrm{H}-4,60-62$ & 11.90 & 0.341 & 8.41 & Barren & & $*$ \\
\hline $2 \mathrm{H}-5,60-62$ & 13.40 & 0.384 & 10.17 & Few & Moderate & \\
\hline $2 \mathrm{H}-6,60-62$ & 14.90 & 0.427 & 9.06 & Barren & & * \\
\hline $2 \mathrm{H}-7,60-62$ & 16.40 & 0.469 & 5.79 & Few & Moderate & $\mathrm{D}, *$ \\
\hline $2 \mathrm{H}-\mathrm{CC}$ & 16.30 & 0.467 & & Rare & Moderate & \\
\hline $3 \mathrm{H}-1,60-62$ & 16.90 & 0.484 & 8.48 & Few & Poor & 2 IR, trissocyclids \\
\hline $3 \mathrm{H}-2,60-62$ & 18.40 & 0.527 & 7.97 & Common & Moderate & D \\
\hline $3 \mathrm{H}-3,60-62$ & 19.90 & 0.570 & 11.12 & Rare & Moderate & $\mathrm{D}, *$ \\
\hline $3 \mathrm{H}-4,60-62$ & 21.40 & 0.613 & 8.64 & Rare & Moderate & $\mathrm{D}, * 2 \mathrm{IR}$ \\
\hline $3 \mathrm{H}-5,59-61$ & 22.89 & 0.655 & 4.87 & Barren & & * \\
\hline $3 \mathrm{H}-6,60-62$ & 24.40 & 0.699 & 8.71 & Barren & & \\
\hline $3 \mathrm{H}-7,60-62$ & 25.90 & 0.742 & 10.97 & Rare & Poor & \\
\hline $3 \mathrm{H}-\mathrm{CC}$ & 25.80 & 0.739 & & Rare & Moderate & \\
\hline $4 \mathrm{H}-1,59-61$ & 26.39 & 0.756 & 12.23 & Barren & & \\
\hline $4 \mathrm{H}-2,59-61$ & 27.89 & 0.800 & 7.92 & Few & Good & D \\
\hline $4 \mathrm{H}-3,59-62$ & 29.39 & 0.844 & 8.36 & Few & Moderate & 2 IR \\
\hline $4 \mathrm{H}-4,59-61$ & 30.89 & 0.889 & 7.35 & Few & Moderate & High IR \\
\hline $4 \mathrm{H}-5,59-61$ & 32.39 & 0.933 & 8.50 & Common & Moderate & $2 \mathrm{IR}$ \\
\hline $4 \mathrm{H}-6,59-62$ & 33.89 & 0.977 & 10.42 & Common & Moderate to poor & D \\
\hline $4 \mathrm{H}-7,58-60$ & 35.38 & 1.024 & 14.66 & Rare & Moderate to poor & \\
\hline $4 \mathrm{H}-\mathrm{CC}$ & 35.30 & 1.021 & & Abundant & Good & \\
\hline $5 \mathrm{H}-1,60-63$ & 35.90 & 1.040 & 10.72 & Few & Moderate & D \\
\hline $5 \mathrm{H}-2,62-65$ & 37.42 & 1.088 & 16.83 & Barren & & $*$ \\
\hline $5 \mathrm{H}-3,60-63$ & 38.90 & 1.135 & 15.50 & Barren & & * \\
\hline $5 \mathrm{H}-4,59-61$ & 40.39 & 1.182 & 15.75 & Rare & Poor & * \\
\hline $5 \mathrm{H}-5,58-60$ & 41.88 & 1.229 & 12.96 & Rare & Poor & $*$ \\
\hline $5 \mathrm{H}-6,63-65$ & 43.43 & 1.278 & 15.91 & Barren & & $\mathrm{D}, *$ \\
\hline $5 \mathrm{H}-\mathrm{CC}$ & 44.80 & 1.322 & & Rare & Moderate & \\
\hline $6 \mathrm{H}-1,59-61$ & 45.39 & 1.340 & 8.94 & Few & Moderate & \\
\hline $6 \mathrm{H}-2,59-61$ & 46.89 & 1.388 & 14.13 & Few & Good & \\
\hline $6 \mathrm{H}-3,71-74$ & 48.51 & 1.439 & 15.97 & Barren & & $*$ \\
\hline $6 \mathrm{H}-4,62-64$ & 49.92 & 1.484 & 12.82 & Barren & & * \\
\hline $6 \mathrm{H}-5,59-61$ & 51.39 & 1.530 & 11.81 & Few & Moderate & $*$ \\
\hline $6 \mathrm{H}-6,59-61$ & 52.89 & 1.577 & 19.59 & Barren & & * \\
\hline $6 \mathrm{H}-\mathrm{CC}$ & 54.30 & 1.622 & & Barren & & \\
\hline $7 \mathrm{H}-1,60-62$ & 54.90 & 1.641 & 10.98 & Rare & Moderate & * \\
\hline $7 \mathrm{H}-2,60-62$ & 56.40 & 1.697 & 13.84 & Barren & & \\
\hline $7 \mathrm{H}-3,60-62$ & 57.90 & 1.758 & 10.92 & Rare & Moderate & \\
\hline $7 \mathrm{H}-4,60-62$ & 59.40 & 1.819 & 10.87 & Rare & Poor & \\
\hline $7 \mathrm{H}-5,60-62$ & 60.90 & 1.880 & 9.38 & Few & Moderate to poor & \\
\hline $7 \mathrm{H}-6,60-62$ & 62.40 & 1.941 & 8.38 & Few & Moderate & \\
\hline $7 \mathrm{H}-\mathrm{CC}$ & 63.80 & 1.998 & & Common & Good & \\
\hline $8 \mathrm{H}-1,60-62$ & 64.40 & 2.022 & 7.78 & Few & Poor & \\
\hline $8 \mathrm{H}-2,60-62$ & 65.90 & 2.083 & 12.95 & Common & Moderate & \\
\hline $8 \mathrm{H}-3,60-62$ & 67.40 & 2.144 & 10.02 & Common & Moderate & \\
\hline $8 \mathrm{H}-4,60-62$ & 68.90 & 2.205 & 6.52 & Abundant & Moderate & $\mathrm{f}$ \\
\hline $8 \mathrm{H}-5,59-61$ & 70.40 & 2.266 & 5.46 & Abundant & Moderate & $\mathrm{f}$ \\
\hline $8 \mathrm{H}-6,60-62$ & 71.90 & 2.328 & 8.48 & Few & Moderate & $\mathrm{f}, 2 \mathrm{IR}$ \\
\hline $8 \mathrm{H}-\mathrm{CC}$ & 73.30 & 2.385 & & Abundant & Good & \\
\hline $9 \mathrm{H}-1,59-61$ & 73.89 & 2.409 & 6.59 & Abundant & Good & f \\
\hline $9 \mathrm{H}-2,59-61$ & 75.39 & 2.470 & 11.24 & Abundant & Moderate & f \\
\hline $9 \mathrm{H}-3,59-61$ & 76.89 & 2.516 & 7.79 & Common & Moderate & \\
\hline $9 \mathrm{H}-4,59-61$ & 78.39 & 2.561 & 9.22 & Abundant & Good & \\
\hline $9 \mathrm{H}-5,59-61$ & 79.89 & 2.607 & 12.66 & Common & Moderate & \\
\hline $9 \mathrm{H}-6,59-61$ & 81.39 & 2.653 & 9.40 & Abundant & Moderate & \\
\hline $9 \mathrm{H}-7,59-61$ & 82.89 & 2.699 & 8.91 & Abundant & Moderate & \\
\hline $9 \mathrm{H}-\mathrm{CC}$ & 82.80 & 2.696 & & Abundant & Good & \\
\hline $10 \mathrm{H}-1,60-62$ & 83.40 & 2.715 & 10.84 & Abundant & Good & * \\
\hline $10 \mathrm{H}-2,60-62$ & 84.90 & 2.761 & 9.51 & Abundant & Moderate & \\
\hline $10 \mathrm{H}-3,60-62$ & 86.40 & 2.806 & 6.71 & Common & Moderate & \\
\hline $10 \mathrm{H}-4,60-62$ & 87.90 & 2.852 & 15.16 & Abundant & Good & \\
\hline
\end{tabular}

${ }^{\mathrm{a}} \mathrm{f}$ indicates presence of floaters, D indicates sample comes from a dark layer, * indicates presence of sand-sized grains, and $2 \mathrm{IR}$ indicates that radiolarians are present with two states of refractive index (IR). 
Table 3. Late Pliocene and Pleistocene age Hole 795A radiolarian samples with estimates of radiolarian abundance and preservation.

\begin{tabular}{|c|c|c|c|c|c|c|}
\hline \multirow{2}{*}{$\begin{array}{l}\text { Core, section, } \\
\text { interval }(\mathrm{cm})\end{array}$} & \multirow{2}{*}{$\begin{array}{l}\text { Depth } \\
\text { (mbsf) }\end{array}$} & \multirow{2}{*}{$\begin{array}{l}\text { Age } \\
\text { (Ma) }\end{array}$} & \multirow{2}{*}{$\begin{array}{l}\text { Slide weight } \\
\text { (g) }\end{array}$} & \multicolumn{2}{|c|}{ Radiolarians } & \multirow[b]{2}{*}{ Comments ${ }^{\mathrm{a}}$} \\
\hline & & & & Abundance & Preservation & \\
\hline \multicolumn{7}{|l|}{$127-795 \mathrm{~A}-$} \\
\hline 1H-CC & 9.30 & 0.194 & & Rare & Good & \\
\hline $2 \mathrm{H}-\mathrm{CC}$ & 18.80 & 0.392 & & Rare to few & Moderate & \\
\hline $3 \mathrm{H}-\mathrm{CC}$ & 28.30 & 0.590 & & Abundant & Good & \\
\hline $4 \mathrm{H}-\mathrm{CC}$ & 37.80 & 0.780 & & Rare & Moderate & \\
\hline $5 \mathrm{H}-\mathrm{CC}$ & 47.30 & 0.950 & & Abundant & Good & \\
\hline $6 \mathrm{H}-\mathrm{CC}$ & 56.80 & 1.120 & & Few & Moderate & \\
\hline 7H-CC & 66.30 & 1.290 & & Rare & Poor & \\
\hline $8 \mathrm{H}-\mathrm{CC}$ & 75.80 & 1.460 & & Few & Moderate & \\
\hline 9H-CC & 85.30 & 1.630 & & Rare & Poor & \\
\hline $10 \mathrm{H}-\mathrm{CC}$ & 94.80 & 1.761 & & Rare & Moderate & \\
\hline $11 \mathrm{H}-\mathrm{CC}$ & 104.30 & 1.884 & & Few & Moderate & \\
\hline $12 \mathrm{H}-1,70-72$ & 105.00 & 1.893 & 13.01 & Common & Moderate & $* 2 \mathrm{IR}$ \\
\hline $12 \mathrm{H}-2,70-72$ & 106.50 & 1.912 & 9.90 & Few & Moderate & \\
\hline $12 \mathrm{H}-3,70-72$ & 108.00 & 1.932 & 11.05 & Few & Good & \\
\hline $12 \mathrm{H}-4,70-72$ & 109.50 & 1.951 & 8.19 & Few & Good & f \\
\hline $12 \mathrm{H}-5,70-72$ & 111.00 & 1.971 & 9.82 & Few & Moderate & f, 2 IR \\
\hline $12 \mathrm{H}-6,70-72$ & 112.50 & 1.990 & 11.66 & Common & Moderate & f. 2 IR \\
\hline $12 \mathrm{H}-\mathrm{CC}$ & 113.80 & 2.007 & & Common & Moderate & \\
\hline $13 \mathrm{H}-1,74-76$ & 114.54 & 2.016 & 15.85 & Few & Moderate & \\
\hline $13 \mathrm{H}-2,74-76$ & 116.04 & 2.036 & 12.58 & Common & Good & f \\
\hline $13 \mathrm{H}-3,74-76$ & 117.54 & 2.055 & 15.35 & Few & Moderate & f. 2 IR \\
\hline $13 \mathrm{H}-4,74-76$ & 119.04 & 2.075 & 11.22 & Few & Moderate & f, 2 IR \\
\hline $13 \mathrm{H}-5,74-76$ & 120.54 & 2.094 & 13.93 & Common & Moderate & f \\
\hline $13 \mathrm{H}-6,74-76$ & 122.04 & 2.114 & 10.52 & Common & Good & f \\
\hline $13 \mathrm{H}-7,51-53$ & 123.21 & 2.129 & 15.51 & Common & Good & f \\
\hline $13 \mathrm{H}-\mathrm{CC}$ & 123.30 & 2.130 & & Common & Moderate & \\
\hline $14 \mathrm{H}-2,73-75$ & 125.53 & 2.159 & 12.27 & Common & Moderate & $2 \mathrm{IR}$ \\
\hline $14 \mathrm{H}-3,73-75$ & 127.03 & 2.178 & 13.88 & Common & Moderate & f \\
\hline $14 \mathrm{H}-4,73-75$ & 128.53 & 2.198 & 11.88 & Abundant & Moderate & $f$ \\
\hline $14 \mathrm{H}-5,73-75$ & 130.03 & 2.217 & 13.45 & Abundant & Good & $f$ \\
\hline $14 \mathrm{H}-6,73-75$ & 131.53 & 2.236 & 13.16 & Abundant & Moderate & f \\
\hline $14 \mathrm{H}-7,51-53$ & 132.81 & 2.253 & 16.25 & Abundant & Moderate & $f$ \\
\hline $14 \mathrm{H}-\mathrm{CC}$ & 132.80 & 2.253 & & Abundant & Good & \\
\hline $15 \mathrm{H}-1,73-75$ & 133.53 & 2.262 & 8.85 & Common & Good & f \\
\hline $15 \mathrm{H}-2,73-75$ & 135.03 & 2.282 & 9.72 & Abundant & Good & f \\
\hline $15 \mathrm{H}-3,73-75$ & 136.53 & 2.301 & 6.84 & Few & Good & $f$ \\
\hline $15 \mathrm{H}-4,73-75$ & 138.03 & 2.320 & 6.88 & Rare & Poor & f, 2 IR \\
\hline $15 \mathrm{H}-5,73-75$ & 139.53 & 2.340 & 9.97 & Few & Good & f \\
\hline $15 \mathrm{H}-6,73-75$ & 141.03 & 2.359 & 9.17 & Common & Moderate to good & $f$ \\
\hline $15 \mathrm{H}-7,51-53$ & 142.31 & 2.376 & 11.14 & Few & Good & $f$ \\
\hline $15 \mathrm{H}-\mathrm{CC}$ & 142.30 & 2.376 & & Abundant & Moderate & \\
\hline $16 \mathrm{H}-1,73-75$ & 143.03 & 2.385 & 9.42 & Abundant & Good & $\mathrm{f}$ \\
\hline $16 \mathrm{H}-2,73-75$ & 144.53 & 2.405 & 12.12 & Few & Moderate & f \\
\hline $16 \mathrm{H}-3,73-75$ & 146.03 & 2.424 & 11.72 & Common & Moderate & \\
\hline $16 \mathrm{H}-4,74-76$ & 147.54 & 2.444 & 11.73 & Common & Moderate & $\mathrm{f}$ \\
\hline $16 \mathrm{H}-\mathrm{CC}$ & 151.80 & 2.499 & & Common & Good & \\
\hline $17 \mathrm{H}-\mathrm{CC}$ & 151.90 & 2.500 & & Common & Good & \\
\hline
\end{tabular}

${ }^{\mathrm{a}} \mathrm{f}$ indicates presence of floaters, * indicates presence of sand-sized grains, and $2 \mathrm{IR}$ indicates that radiolarians are present with two states of refractive index (IR).

obtained from these sediments provides an excellent opportunity to study the preservation of radiolarians. The preservation of radiolarian assemblages from Japan Sea Leg 127 sediments represents several rather extreme examples of preservation state. The radiolarians from the deeper, opal-CT sediments are rather poorly preserved, however, the state of poor preservation of radiolarians found in the opal-CT sediments is quite different from other states of poor preservation found in sediments of the Japan Sea from above the opal-A/opal-CT transition, because they were preserved by coatings of opal-CT. Radiolarians from the Miocene and Pliocene diatomaceous sediments are relatively well preserved, and display few visible signs of dissolution; however, their preservation and abundance records are somewhat obscured because they are outnumbered in the radiolarian slides by high abundances of the diatom Coscinodiscus marginatus. The Pleistocene radiolarian assemblages in Japan Sea ODP Leg 127 sediments are in general the most poorly preserved; however, there is a small minority of samples from sediments younger than the opal dissolution transition zone which contain well-preserved, abundant radiolarian assemblages (Tables 2, 3, and 4).

Observations on and comparisons between the preservation of radiolarians in the late Pliocene and Pleistocene samples from Sites 794, 795, and 797 reveal that certain types of radiolarians from Japan Sea sediments are solution resistant. The two types of most dissolution-resistant radiolarians include morphotypes (PI. 1, Figs. 4 and 5, and Pl. 2, Fig. 2) of trissocyclids (Goll, 1968), and a radiolarian identified as Peripyramis species (Pl. 2, Fig. 3, and Pl. 4, Fig. 5). In samples where only these species were found, tests displayed visible signs of dissolution (Pl. 1, Fig. 4), which made identifications of species impossible, but confirmed the notion that concentrations of these species in samples are indicators of high levels of dissolution. The dissolution-resistant trissocyclids of the Japan Sea are very similar in form to the spyrid radiolarians identified as dissolution-resistant forms by Holdsworth and Harker (1975), which supports the hypothesis that trissocyclid radiolarians are solution-resistant forms. It is interesting to note that collosphaerids, which were also 
Table 4. Late Pliocene and Pleistocene age Hole 797B radiolarian samples with estimates of radiolarian abundance and preservation.

\begin{tabular}{|c|c|c|c|c|c|c|}
\hline \multirow{2}{*}{$\begin{array}{l}\text { Core, section, } \\
\text { interval }(\mathrm{cm})\end{array}$} & \multirow{2}{*}{$\begin{array}{l}\text { Depth } \\
\text { (mbsf) }\end{array}$} & \multirow{2}{*}{$\begin{array}{l}\text { Age } \\
\text { (Ma) }\end{array}$} & \multirow{2}{*}{$\begin{array}{l}\text { Slide weight } \\
\text { (g) }\end{array}$} & \multicolumn{2}{|c|}{ Radiolarians } & \multirow{2}{*}{ Comments $^{\mathrm{a}}$} \\
\hline & & & & Abundance & Preservation & \\
\hline \multicolumn{7}{|l|}{ 127-797B- } \\
\hline $1 \mathrm{H}-\mathrm{CC}$ & 5.90 & 0.130 & & Common & Moderate & \\
\hline $2 \mathrm{H}-1,118-120$ & 7.08 & 0.156 & 6.91 & Rare & Poor & 2 IR \\
\hline $2 \mathrm{H}-2,118-120$ & 8.58 & 0.189 & 8.16 & Rare & Moderate & $*$ \\
\hline $2 \mathrm{H}-3,131-133$ & 10.11 & 0.222 & 11.41 & Rare & Moderate & *2 IR \\
\hline $2 \mathrm{H}-4,118-120$ & 11.58 & 0.255 & 10.31 & Rare & Moderate & 2 IR \\
\hline $2 \mathrm{H}-5,118-120$ & 13.08 & 0.287 & 6.65 & Common & Moderate & $2 \mathrm{IR}$ \\
\hline $2 \mathrm{H}-6,118-120$ & 14.58 & 0.320 & 11.25 & Barren & & $*$ \\
\hline $2 \mathrm{H}-\mathrm{CC}$ & 15.40 & 0.338 & & Rare & Moderate & \\
\hline $3 \mathrm{H}-1,117-119$ & 16.57 & 0.364 & 7.02 & Rare & Moderate & D, 2 IR \\
\hline $3 \mathrm{H}-2,117-119$ & 18.07 & 0.397 & 4.85 & Common & Poor & f, 2 IR \\
\hline $3 \mathrm{H}-3,117-119$ & 19.57 & 0.430 & 8.85 & Barren & & $\mathrm{f}, *$ \\
\hline $3 \mathrm{H}-4,117-119$ & 21.07 & 0.463 & 6.74 & Common & Moderate & f, D \\
\hline $3 \mathrm{H}-5,111-113$ & 22.51 & 0.495 & 7.02 & Rare & Moderate & $2 \mathrm{IR}$ \\
\hline $3 \mathrm{H}-6,117-119$ & 24.07 & 0.529 & 9.62 & Rare & Poor & f, $*$ \\
\hline $3 \mathrm{H}-\mathrm{CC}$ & 24.90 & 0.547 & & Rare & Good & \\
\hline $4 \mathrm{H}-1,118-120$ & 26.08 & 0.573 & 5.02 & Common & Poor & High IR \\
\hline $4 \mathrm{H}-2,118-120$ & 27.58 & 0.606 & 6.49 & Abundant & Poor & f, High IR, trissocyclids \\
\hline $4 \mathrm{H}-3,118-120$ & 29.08 & 0.639 & 7.05 & Common & Poor & f, High IR \\
\hline $4 \mathrm{H}-4,118-120$ & 30.58 & 0.672 & 6.84 & Abundant & Good & f \\
\hline $4 \mathrm{H}-5,118-120$ & 32.08 & 0.705 & 5.89 & Common & Poor to moderate & f, *High IR \\
\hline $4 \mathrm{H}-6,118-120$ & 33.58 & 0.738 & 8.25 & Common & Moderate & f, D trissocyclids \\
\hline $4 \mathrm{H}-\mathrm{CC}$ & 34.40 & 0.756 & & Rare & Moderate & \\
\hline $5 \mathrm{H}-1,118-120$ & 35.58 & 0.782 & 7.24 & Barren & & f, * \\
\hline $5 \mathrm{H}-2,118-120$ & 37.08 & 0.815 & 8.48 & Rare & Poor & f, D High IR \\
\hline $5 \mathrm{H}-3,118-120$ & 38.58 & 0.848 & 8.88 & Common & Moderate & 2 IR \\
\hline $5 \mathrm{H}-4,118-120$ & 40.08 & 0.881 & 7.22 & Few & Poor & f, High IR \\
\hline $5 \mathrm{H}-5,118-120$ & 41.58 & 0.914 & 11.63 & Rare & Poor & *High IR \\
\hline $5 \mathrm{H}-6,118-120$ & 43.08 & 0.947 & 11.79 & Rare & Moderate & $*$ \\
\hline $5 \mathrm{H}-\mathrm{CC}$ & 43.90 & 0.965 & & Common & Good & \\
\hline $6 \mathrm{H}-1,115-117$ & 45.05 & 0.990 & 10.10 & Rare & Poor & $f, *$ \\
\hline $6 \mathrm{H}-2,118-120$ & 46.58 & 1.024 & 9.12 & Common & Moderate & $\mathrm{f}, *$ \\
\hline $6 \mathrm{H}-3,118-120$ & 48.08 & 1.057 & 5.10 & Common & Moderate & f, D \\
\hline $6 \mathrm{H}-4,110-112$ & 49.58 & 1.090 & 7.86 & Abundant & Moderate & $f$ \\
\hline $6 \mathrm{H}-5,118-120$ & 51.08 & 1.123 & 6.06 & Rare & Moderate & \\
\hline $6 \mathrm{H}-6,118-120$ & 52.58 & 1.156 & 13.02 & Rare & Moderate & $f, *$ \\
\hline $6 \mathrm{H}-\mathrm{CC}$ & 53.40 & 1.174 & & Abundant & Good & \\
\hline $7 \mathrm{H}-1,118-120$ & 54.58 & 1.200 & 11.88 & Few & Moderate & f, * \\
\hline $7 \mathrm{H}-2,118-120$ & 56.08 & 1.233 & 9.33 & Rare & Good & * \\
\hline $7 \mathrm{H}-3,117-119$ & 57.57 & 1.265 & 7.47 & Common & Moderate & \\
\hline $7 \mathrm{H}-4,117-119$ & 59.07 & 1.298 & 7.01 & Common & Moderate & \\
\hline $7 \mathrm{H}-5,117-119$ & 60.27 & 1.325 & 8.63 & Rare & Moderate & \\
\hline $7 \mathrm{H}-6,117-119$ & 62.07 & 1.364 & 9.22 & Rare & Poor & \\
\hline 7H-CC & 62.90 & 1.382 & & Abundant & Good & \\
\hline $8 \mathrm{H}-1,118-120$ & 64.08 & 1.408 & 8.49 & Abundant & Good & f \\
\hline $8 \mathrm{H}-2,118-120$ & 65.58 & 1.441 & 11.72 & Rare & Poor & \\
\hline $8 \mathrm{H}-3,118-120$ & 67.08 & 1.474 & 12.11 & Rare & Moderate & * \\
\hline $8 \mathrm{H}-4,118-120$ & 68.58 & 1.507 & 8.60 & Abundant & Good & \\
\hline $8 \mathrm{H}-5,118-120$ & 70.08 & 1.540 & 10.13 & Rare & Moderate & $*$ \\
\hline $8 \mathrm{H}-6,118-120$ & 71.58 & 1.573 & 8.75 & Common & Moderate & \\
\hline $8 \mathrm{H}-\mathrm{CC}$ & 72.40 & 1.591 & & Common & Good & \\
\hline $9 \mathrm{H}-1,118-120$ & 73.58 & 1.617 & 8.75 & Rare & Moderate & $*$ \\
\hline $9 \mathrm{H}-2,118-120$ & 75.08 & 1.650 & 13.70 & Rare & Moderate & $*$ \\
\hline $9 \mathrm{H}-3,118-120$ & 76.58 & 1.683 & 9.29 & Rare & Poor & \\
\hline $9 \mathrm{H}-5,118-120$ & 79.58 & 1.749 & 10.68 & Rare & Poor & \\
\hline $9 \mathrm{H}-6,118-120$ & 81.08 & 1.782 & 8.29 & Few & Moderate & \\
\hline $9 \mathrm{H}-\mathrm{CC}$ & 81.90 & 1.800 & & Common & Moderate & \\
\hline $10 \mathrm{H}-1,118-120$ & 83.08 & 1.824 & 11.73 & Common & Moderate & \\
\hline $10 \mathrm{H}-2,118-120$ & 84.58 & 1.854 & 11.72 & Abundant & Moderate & $2 \mathrm{IR}$ \\
\hline $10 \mathrm{H}-3,118-120$ & 86.08 & 1.884 & 16.53 & Few & Moderate & \\
\hline $10 \mathrm{H}-4,118-120$ & 87.58 & 1.914 & 7.92 & Common & Moderate & \\
\hline $10 \mathrm{H}-5,118-120$ & 89.08 & 1.944 & 7.93 & Common & Moderate & \\
\hline $10 \mathrm{H}-6,118-120$ & 90.58 & 1.975 & 11.66 & Few & Moderate & \\
\hline $10 \mathrm{H}-\mathrm{CC}$ & 91.40 & 1.991 & & Few & Moderate & \\
\hline $11 \mathrm{H}-1,118-120$ & 92.58 & 2.015 & 8.07 & Common & Moderate & \\
\hline $11 \mathrm{H}-2,118-120$ & 94.08 & 2.045 & 9.10 & Common & Moderate & \\
\hline $11 \mathrm{H}-3,118-120$ & 95.58 & 2.075 & 10.24 & Few & Moderate & f \\
\hline $11 \mathrm{H}-4,118-120$ & 97.08 & 2.105 & 10.68 & Abundant & Moderate & High IR \\
\hline $11 \mathrm{H}-5,118-120$ & 98.58 & 2.136 & 9.46 & Few & Poor & 2 IR \\
\hline $11 \mathrm{H}-6,118-120$ & 100.58 & 2.176 & 8.57 & Common & Moderate & f, trissocyclids \\
\hline $11 \mathrm{H}-\mathrm{CC}$ & 100.90 & 2.182 & & Common & Moderate & \\
\hline
\end{tabular}


Table 4 (continued).

\begin{tabular}{|c|c|c|c|c|c|c|}
\hline \multirow{2}{*}{$\begin{array}{l}\text { Core, section, } \\
\text { interval }(\mathrm{cm})\end{array}$} & \multirow{2}{*}{$\begin{array}{l}\text { Depth } \\
\text { (mbsf) }\end{array}$} & \multirow{2}{*}{$\begin{array}{l}\text { Age } \\
\text { (Ma) }\end{array}$} & \multirow{2}{*}{$\begin{array}{l}\text { Slide weight } \\
\text { (g) }\end{array}$} & \multicolumn{2}{|c|}{ Radiolarians } & \multirow[b]{2}{*}{ Comments $^{\mathrm{a}}$} \\
\hline & & & & Abundance & Preservation & \\
\hline $12 \mathrm{H}-1,117-119$ & 102.70 & 2.218 & 6.80 & Abundant & Good & \\
\hline $12 \mathrm{H}-2,117-119$ & 103.57 & 2.236 & 6.66 & Abundant & Moderate to poor & f \\
\hline $12 \mathrm{H}-3,117-119$ & 105.07 & 2.266 & 7.45 & Abundant & Good & f \\
\hline $12 \mathrm{H}-4,113-115$ & 106.53 & 2.296 & 9.77 & Abundant & Moderate to poor & f, trissocyclids \\
\hline $12 \mathrm{H}-5,117-119$ & 108.07 & 2.327 & 5.29 & Abundant & Good & \\
\hline $12 \mathrm{H}-6,117-119$ & 109.57 & 2.357 & 7.40 & Abundant & Good & f \\
\hline $12 \mathrm{H}-\mathrm{CC}$ & 110.40 & 2.373 & & Common & Good & \\
\hline $13 \mathrm{H}-1,118-120$ & 111.58 & 2.397 & 7.40 & Abundant & Good & \\
\hline $13 \mathrm{H}-2,118-120$ & 113.08 & 2.427 & 8.88 & Common & Moderate & trissocyclids \\
\hline
\end{tabular}

" $\mathrm{f}$ indicates presence of floaters, D indicates sample comes from a dark layer, * indicates presence of sand-sized grains, and 2 IR indicates that radiolarians are present with two states of refractive index (IR).

found concentrated with trissocyclid radiolarians in the dissolved assemblages described by Holdsworth and Harker (1975), were not found in any samples from the Leg 127 sites. Because collosphaerid radiolarians may be indicative of more oligotrophic conditions, the absence of them from Japan Sea sediments suggests that the Japan Sea has always been highly productive. Also, because collosphaerids live in colonies that can grow quite large, it is possible that the absence of collosphaerid radiolarians from Japan Sea sediments is due to oceanographic conditions unsuitable for the survival of these species, such as high levels of grazing and/or a well-mixed water column.

Another important preservational indicator of radiolarians of Leg 127 sediments is the radiolarian refractive index. Although it is not clear what changes in the refractive index of radiolarians record, samples from Leg 127 that contain radiolarians with high indices of refraction, typically also contain radiolarians that show visible signs of dissolution. Differences in refractive index are easily observed in radiolarians. Radiolarians with a high index of refraction appear ghostly because their index of refraction is closer to that of Canada Balsam (Pl. 1, Fig. 8), whereas radiolarians with a normal or lower index of refraction appear darker. Typically, when radiolarians in a sample display two states of index of refraction (e.g., Pl. 1, Fig. 6), reworking may be inferred because older radiolarians commonly have a lower index of refraction. However, the occurrence of two states of index of refraction within a sample does not necessarily imply that reworking has occurred. In fact, some single test radiolarian specimens within Japan Sea samples with assemblages that displayed two refractive index states also display two states of index of refraction.

Close inspections of specimen that display two states of refractive index (IR) revealed that although the fossils are still relatively complete, the high-IR portions of some of the tests are slightly thinner, indicating that the change to a higher index of refraction accompanies and probably precedes dissolution. Because many radiolarians from samples that display two states of IR display obvious signs of dissolution such as pitting, thinning of connecting bars, reduction of lattice "lace," etc., but do not necessarily display high IR values, it is likely that dissolution occurs after deposition of the radiolarians either at the sediment/water interface or within the sediment column. Because skeletal thinning will make the skeletons of radiolarians more fragile, radiolarians that start dissolving within the water column are more likely to become fragmented due to the rigors of transport and deposition than are those that began dissolving within the sediments.

\section{Reworking}

The distribution of reworked radiolarians in sediments cored during Leg 127 is a good indicator of sediment transport and tectonically induced deposits. Reworking of radiolarians was lowest in the sediments of Site 794, and was more common at Sites 795 and 797 (Tamaki, Pisciotto, Allan, et al., 1990). Relatively low amounts of reworked radiolarians were identified in Site 796 sediments; however, the presence of sands and sediment slumping obscures the radiolarian record at this site. For this reason no additional samples were processed from Site 796 cores for this study. Radiolarian reworking is confirmed in Pliocene and Pleistocene sediments where Miocene forms are identified and also in sediments where there are abundant fragmented radiolarians (PI. 2, Figs. 1 and 2). Commonly, older reworked radiolarians appear thickened and have a lower index of refraction than the rest of the radiolarians in the assemblage. The most common reworked radiolarians were usually species from the genera Stichocorys or Cyrtocapsella (Pl. 2, Fig. 4). Some cycladophorid radiolarians most like Anthocorys akitaensis looked suspiciously reworked in several samples due to slightly lower apparent index of refraction.

\section{RADIOLARIAN STRATIGRAPHIC INDICATORS OF JAPAN SEA SEDIMENTS}

Because of their continuous nature, the sites cored during Leg 127 can be used to construct a reference section for radiolarians from Japan. Therefore, an objective of this radiolarian paleontology and stratigraphy study is to precisely identify the sequence of radiolarian biostratigraphic events of the Japan Sea. Once the radiolarian stratigraphy is clearly established and age models for Leg 127 sediments are refined, the radiolarian stratigraphy can be used to help decipher the depositional history of basins of the Japan Sea where radiolarians are found. However, the identification of stratigraphic datum levels in Leg 127 Japan Sea sediments is complicated by poor preservation in the youngest and oldest sediments recovered, by radiolarian sample contamination by the diatom Coscinodiscus marginatus in Miocene and Pliocene sediments, by incomplete inventories and taxonomies of Japan Sea radiolarian species, and by evolutionary changes throughout the sections examined.

The radiolarian stratigraphy of the Japan Sea is also hard to tie directly to standard zonations from other parts of the Pacific because Pacific indicator species do not occur or occur sporadically in the sediments recovered on Leg 127 . The radiolarian stratigraphy from the Leg 127 sites can be tied to the radiolarian zonations set up for middle Miocene to Pliocene sedimentary deposits of Japan by Nakaseko and Sugano (1973). This zonation is very useful because the boundaries of the zones are defined by only a few species and the ranges of 36 taxa that range between the middle Miocene and Pliocene are traced through these zones. Although indicator species from several genera such as Didymocyrtis, Calocycletta, or Lithopera were not found in the Leg 127 sediments, the fact that they are present in deposits on Japan allows for some correlation with radiolarian assemblages assigned to North Pacific zonations.

Radiolarian zonations set up for Japan Sea sediments (Nakaseko and Sugano, 1973, Tamaki, Pisciotto, Allan, et al., 1990) will not be used to describe the Leg 127 sites examined in this study, because the stratigraphic resolution of the zonations is rather low, and the poor preservation of the late Pliocene and Pleistocene sediments prohibits the exact determination of zonal boundaries. Instead, radiolarian stratigraphy determined in this study is used to examine the correla- 
Table 5. Samples from Hole 794A containing subtropical radiolarians.

\begin{tabular}{|c|c|c|c|c|c|c|}
\hline \multirow{2}{*}{$\begin{array}{l}\text { Core, section, } \\
\text { interval }(\mathrm{cm})\end{array}$} & \multirow{2}{*}{$\begin{array}{l}\text { Depth } \\
\text { (mbsf) }\end{array}$} & \multirow{2}{*}{$\begin{array}{l}\text { Age } \\
(\mathrm{Ma})\end{array}$} & \multirow{2}{*}{$\begin{array}{l}\text { Slide weight } \\
\text { (g) }\end{array}$} & \multicolumn{2}{|c|}{ Radiolarians } & \multirow[b]{2}{*}{ Comments } \\
\hline & & & & Abundance & Preservation & \\
\hline \multicolumn{7}{|l|}{$127-794 \mathrm{~A}-$} \\
\hline $1 \mathrm{H}-4,60-62$ & 5.10 & 0.146 & 8.18 & Rare & Poor & \\
\hline IH-5, 55-57 & 6.55 & 0.188 & 10.44 & Rare & Moderate & * \\
\hline $2 \mathrm{H}-2,60-62$ & 8.90 & 0.255 & 5.59 & Few & Moderate & * \\
\hline $4 \mathrm{H}-4,59-61$ & 30.89 & 0.889 & 7.35 & Few & Moderate & \\
\hline $4 \mathrm{H}-5,59-61$ & 32.39 & 0.933 & 8.50 & Common & Moderate & \\
\hline $4 \mathrm{H}-6,59-62$ & 33.89 & 0.977 & 10.42 & Common & Moderate to poor & \\
\hline $5 \mathrm{H}-1,60-63$ & 35.90 & 1.040 & 10.72 & Few & Moderate & \\
\hline $6 \mathrm{H}-1,59-61$ & 45.39 & 1.340 & 8.94 & Few & Moderate & \\
\hline $6 \mathrm{H}-2,59-61$ & 46.89 & 1.388 & 14.13 & Few & Good & \\
\hline $7 \mathrm{H}-1,60-62$ & 54.90 & 1.641 & 10.98 & Rare & Moderate & $*$ \\
\hline
\end{tabular}

a * indicates samples containing sand-sized grains.

tions and relative timing of the events among Japan Sea Sites 794, 795, and 797. Although Ling (1975) examined radiolarians in DSDP Leg 31 sediments and documented the levels of several biostratigraphic events, no previous studies exist in which radiolarian firstoccurrence (FO) and last-occurrence (LO) event stratigraphy from Japan Sea or marine sediments from Japan are tied to a paleomagnetic time scale. In addition, most of the radiolarian datum levels identified in Japan Sea sediments have not been assigned ages in studies of Pacific sediments. Therefore, radiolarian stratigraphy cannot be used to define and refine age models for the Japan Sea Leg 127 sites. The Leg 127 Japan Sea sites can, however, be used as reference sections, and radiolarian datum levels can be assigned ages by interpolating between age control points of other chronological indicators identified in the cores.

Before radiolarian biostratigraphic events can be dated, the sequence and relative reliability of the events must be documented. This is achieved by plotting the 25 first- or last-occurrence events identified in the Japan Sea Leg 127 sediments (Table 1) on depth-depth plots (Fig. 1). In these plots, the sample-spacing uncertainty of the datum levels is represented by boxes, and unreliable or diachronous radiolarian datum levels are identified when they violate apparent lines of correlation between the stratigraphic sections. Lines of correlation are not indicated in Figure 1 because many of the radiolarian datum levels have not been researched in detail and appear to be out of sequence at some of the sites. The best correlation of the radiolarian datum levels from Leg 127 sites occurs between Sites 794 and 797; however an offset of the correlation is found below the FO of Theocalyptra davisiana and the opal dissolution transition zone (Fig. 1). This offset could be indicative of problems in the identification of the LO of Lipmanella sp. aff. $L$. redondoensis, a relative decrease in the sedimentation rate at Site 797, or, an increase in the sedimentation rate at Site 794 in the younger sediments. The gaps in the depth-depth plots between Site 795 and Sites 794 and 797 suggest that the sedimentation rate in the interval corresponding to the range of Lipmanella $\mathrm{sp}$. aff. L redondoensis (175 and $300 \mathrm{~m}$ below seafloor) at Site 795 is significantly higher.

The depth-depth plots that compare the radiolarian stratigraphy of Leg 127 Sites 794, 795, and 797 illustrate that further biostratigraphic analysis is warranted. Until taxonomic uncertainties are resolved and a clear homotaxial pattern is discerned between the three sites, any conclusions about the correlation of the stratigraphic records of these sites is tentative. This preliminary stratigraphic analysis includes illustrations of 14 Japan Sea radiolarian stratigraphic indicators in the plates, and observations on several of these indicator species which are discussed in the following.

The identification of the LO of Clathrocyclas cabrilloensis (Pl. 5, Figs. 7, 8,9, and 10) is complicated by both close affinities with other Cycladophorid morphotypes (Pl. 5, Figs. 4, 5, and 6) and the poor preservation of the Pleistocene assemblages. Despite this, the morphological definition followed for this species is rather robust, and the stratigraphic level of this LO event correlates well among the three sites (Fig. 1).

Because the LO of Stylacontarium acquilonius (Pl. 3, Fig. 2) occurs within the cyclical Pleistocene sediments that exhibit a large variability in preservation, the reliability of this datum level may be suspect. Because it is a rather abundant species, however, its last occurrence is somewhat reliable and appears to correlate among Sites 794,795 , and 797. The LO of the hypothesized ancestor of Stylacontarium acquilonius, Stylacontarium sp. cf. S. acquilonarium, is distinguished from its descendant mainly by the shape of the cortical shell. Because the differences between these two species are very subtle, it is likely that this lineage is equivalent to the species Stylatractus yatsuoensis as described by Nakaseko (1969). The similarities between Stylacontarium acquilonium and Stylatractus yatsuoensis were noted previously by Ling (1975).

The LO of Sphaeropyle robusta (Pl. 3, Fig. 7) and the FO of its descendant, Sphaeropyle langii (PI. 3, Fig. 3), were used as zonal boundary markers for the shipboard reports. Although there are evolutionary intergrades between these two radiolarians in Japan Sea sediments, the first morphotypic appearance of $S$. langii can be easily identified and appears to correlate nicely among sites. The LO of $S$. robusta may not be a reliable stratigraphic indicator because it displays very low abundances in the Pleistocene sediments.

The FO of Theocalyptra davisiana (Pl. 1, Fig. 6, and Pl. 4, Fig. 8) is closely associated with the late Pliocene opal dissolution transition zone (ODTZ). Near the base of the range of Theocalyptra davisiana, ancestral forms and morphotypical intergrades are abundant; therefore, in the Japan Sea, the FO of Theocalyptra davisiana is a morphotypic first appearance. The datum level is probably reliable and synchronous within the Japan Sea sediments, although the occurrence of morphological intergrades deserves more attention.

The LO of Stichocorys peregrina as determined during Leg 127 correlates well between Sites 794 and 797. However, the estimated age for the event is approximately 1 Ma younger in these Japan Sea sites than at Pacific sites, and the range of this event is not consistent with the ranges of the species in the North Pacific. Reworking of this species in the sediments complicates the selection of the level of this last occurrence event.

Sethocyrtis japonica (Pl. 3, Fig. 9), Theocorys redondoensis (Pl. 3, Fig. 5), and Lipmanella sp. aff. Theocorys redondoensis (Pl. 3, Fig. 4) appear to be closely related and may possibly share a common evolutionary lineage. Reynolds (1980) suggested that Sethocyrtis japonica is the ancestor of Theocorys redondoensis. In their overlapping ranges, an intergradation of morphotypes was observed. The complicated evolutionary relationships of these species may explain the apparent 

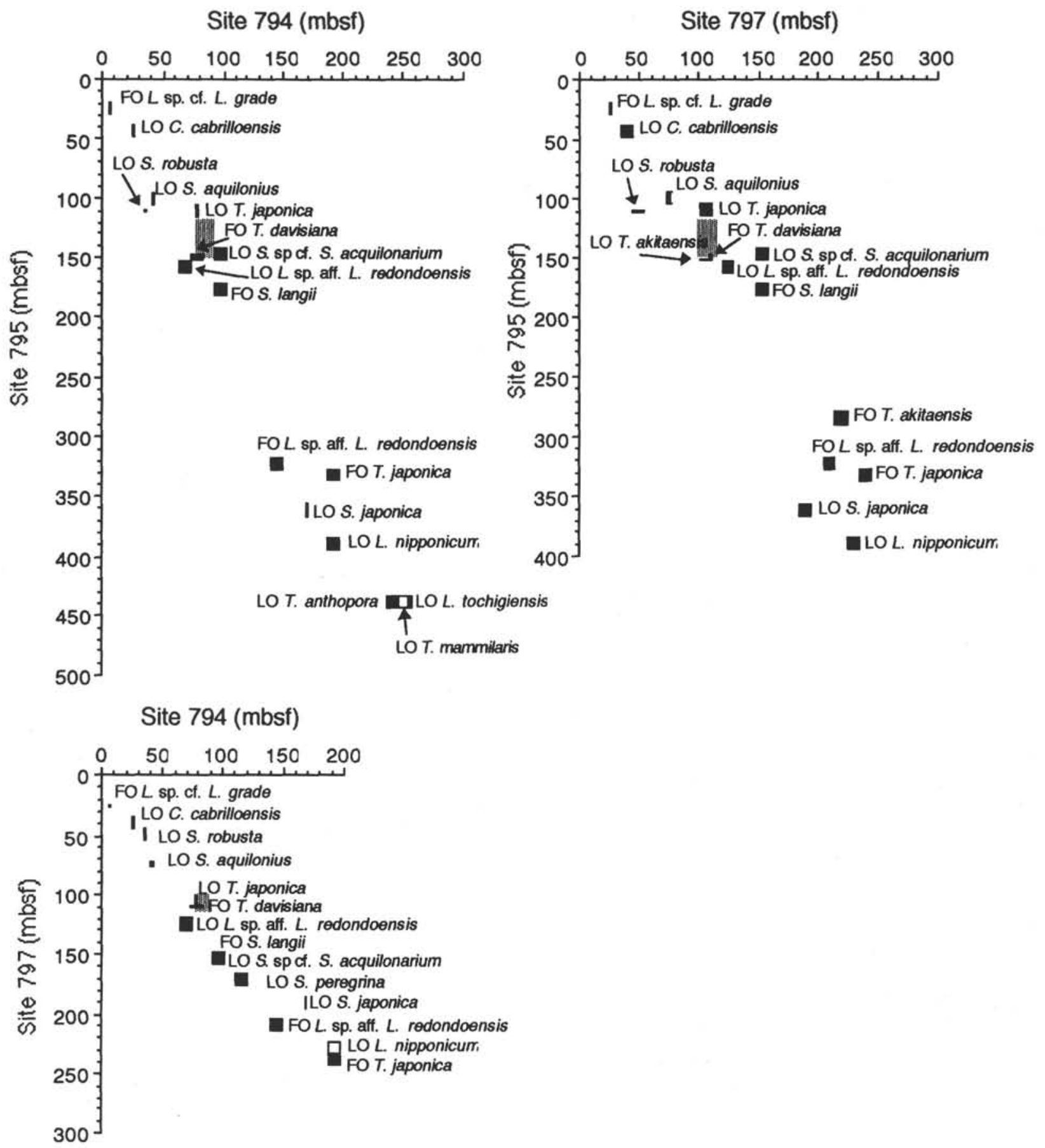

Figure 1. Depth-depth plots comparing the location of radiolarian biostratigraphic events at Leg 127 Sites 794, 795, and 797. The shaded areas denote the opal dissolution transition zone of White and Alexandrovich (this volume), and box sizes represent sample-spacing uncertainties of the datum levels. $\mathrm{LO}$ and FO refer to the last-occurrence and first-occurrence datum levels of the species, respectively.

diachroneity of the LO of S. japonica (Fig. 1) because it may not be an extinction event but rather a pseudo-extinction event, which is more difficult to define.

\section{PALEOCEANOGRAPHIC IMPLICATIONS OF JAPAN SEA RADIOLARIANS}

There are many interesting forms of radiolarians found in the Leg 127 Japan Sea sediments. Many of these have specific ecological tolerances and affinities which can be used to make paleoclimatic and paleoceanographic interpretations. Some Japan Sea radiolarians have undergone morphological changes through time, and can be used to delineate evolutionary histories. A number of noteworthy radiolarian species and lineages with interesting paleoceanographic and evolutionary implications are illustrated and described as follows.

\section{Radiolarian Evidence of a Paleo-Tsushima Current}

Shipboard analyses indicated that radiolarians species with subtropical affinities are not present or are not common in three of the 
sites cored on Leg 127 (Sites 794, 795, and 796), but are common in core-catcher samples from Core 127-797A-8H and above. During Leg 127, Sample 127-796A-6H-CC was the only sample from a site other than Site 797 observed to contain a warmer water assemblage. The subtropical faunal indicators include Tetrapyle octacantha (Pl. 4, Figs. 1 and 2), Didymocyrtis tetrathalamus (PI. 4, Fig. 3), a species belonging to the genus Euchitonia (Pl. 4, Fig. 7), and Spongaster tetras. Shore-based observations indicate that subtropical species are absent from all samples from Hole 795A (Table 3) and are common in samples containing preserved radiolarians from Hole 797B. The first occurrence of these warmer water radiolarian taxa was recorded between Samples 127-797B-9H-6, 118-120 cm, and 127-797B-9H$\mathrm{CC}$, and the sporadic occurrences of these taxa found in samples from Hole 794A are recorded in Table 5.

Presently Site 797 is situated beneath the Tsushima current (an arm of the warm Kuroshio). The occurrence of warm-water (subtropical) fauna in all samples with preserved radiolarians younger than approximately 1.8 Ma from Hole 797B (Table 4) indicates that the Tsushima current probably flowed into the Japan Sea through much of the Pleistocene. However, because the abundance of Tsushima current indicators goes to zero in the Hole 797B barren samples, there may have been intermittent flow of the Tsushima current since this time. In addition, because Tetrapyle octacantha is present in low abundance in the cooler glacial period (Morley et al., 1986), its presence in the Hole 794A samples may record the increased influence of the Tsushima current. These conclusions largely contradict the supposition of Oba et al. (1991) that warmer water from the Tsushima current did not enter the Japan Sea until approximately $10,000 \mathrm{yr}$ ago, but is in agreement with Matoba (1984), who indicated that there was intermittent influx of the Tsushima current since late Pliocene time. If there was a land barrier at the Tsushima Strait throughout most of the Pleistocene (Oba et al., 1991; and Iijima and Tada, 1990), then the occurrence of subtropical radiolarian fauna throughout the Pleistocene would imply that temperatures over Site 797 were much warmer than at the northern sites. It is not likely that warm temperatures could be solely responsible for the persistence of subtropical radiolarians at Site 797, because the influx of the Tsushima current into the Japan Sea is presently responsible for the temperate climate of the region.

\section{Cycladophorid Radiolarians}

Several described taxa compose the Cycladophorid radiolarians in the Leg 127 Japan Sea sediments. These species include Theocalyptra davisiana (Pl. 1, Fig. 6, and Pl. 4, Fig. 8), Cycladophora robusta, Clathrocyclas cabrilloensis, and Anthocorys akitaensis (Pl. 5). Many morphological intergrades were observed in addition to these species, and identification at the species level was hindered by the abundance and diversity of Cycladophorid forms. Cycladophora robusta (PI. 5, Figs. 11 and 12), a probable ancestor of Theocalyptra davisiana, is present in Miocene samples, but its stratigraphic range was not determined. If Cycladophora robusta had an ecological niche similar to its probable descendant Theocalyptra davisiana, the high abundances of Cycladophora robusta observed in samples suggests that cold conditions with a stable upper layer (Morley and Hays, 1983) persisted throughout the entire interval of its range. The fluctuations in the abundance of this radiolarian could imply that these conditions also fluctuated.

\section{Radiolarian Productivity and Paleoclimatic Indicators}

In general, except for the Tsushima current indicators found in Pleistocene samples from Sites 797 and 794, samples examined from the Japan Sea Leg 127 sites contain cool-water radiolarian assemblages. The consistent occurrence of Spongodiscid radiolarians such as Spongotrochus glacialis is indicative that upwelling has persisted in the Japan Sea since the late Miocene. In addition, the negative evidence of the lack of collosphaerid radiolarians in the assemblages, as discussed previously, also indicates that productivity in the Japan Sea was high. There are many other radiolarians found in the Leg 127 Japan Sea sediments that can be used to record fluctuations in water mass properties, such as the species Larnacantha polyacantha, Theocalyptra davisiana, Stylochlamydium venustum, and Tetrapyle octacantha, which define the paleoceanographic factors determined by Morley et al. (1986) from an 80,000-yr-long record from core RC12379 in the Japan Sea. These same radiolarian factors can be used to describe the radiolarian assemblages at Site 797 , but will probably not be very useful at the other sites (Sites 794, 795, and 796) due to the lack of the important species Tetrapyle octacantha.

\section{Radiolarians from Japan Sea Pleistocene Light and Dark Cycles}

An interesting feature of the Leg 127 Japan Sea sediments is the occurrence of distinct light- and dark-color banding. The controls of these Pleistocene light and dark cycles are not well understood and are probably dependent on many factors such as tectonics, sea level, Northern Hemisphere glaciations, deep-water oxygen content, climate, and productivity. Shipboard analyses indicated that the most distinctive feature of the radiolarian assemblages observed in the cyclical Pleistocene sediments of the Japan Sea is high variability in preservation and assemblage composition. Therefore, observations of the details of radiolarian preservation and species composition from the light and dark cycles were made. The occurrence of nonradiolarian material such as sand-sized grains and sponge spicules was also noted.

Radiolarian paleontological samples were not taken for the specific study of light and dark layers and were not obtained at the resolution required to quantify changes in radiolarian assemblages that occur on the time scales of the cycles. By noting if samples from Holes 794A and 797B processed for radiolarians came from dark layers by an examination of the core photos (Tables 2 and 4), a preliminary analysis of the relationship between radiolarian assemblages and sediment banding could be made. No obvious correlation is found between radiolarian assemblage composition or preservation and light and dark color banding. However, because most distinctive dark layers were not sampled, the conclusion that radiolarian assemblage changes can not be related to the light and dark color banding of Leg 127 Japan Sea sediments can not be made. Although no properties observed in radiolarian samples correlate directly with light and dark color banding, it is possible that the variability in the abundance of Theocalyptra davisiana could be correlated to the light and dark cycles. If the correlation of light and dark layers of Japan Sea sediments to the global benthonic oxygen isotope record is correct (Tada, this volume), Theocalyptra davisiana stratigraphy could also be correlated to the cyclicity of light and dark layers because Theocalyptra davisiana records from other deep sea cores have been correlated to records of $\delta^{18} \mathrm{O}$ (Hays et al., 1976, and Morley and Hays, 1979).

\section{Changes in Japan Sea Sill Depths and Isolation of Evolutionary Lineages}

Much more work needs to be performed to determine the evolutionary history of radiolarian assemblages found in Japan Sea sediments. Even with an incomplete understanding of these histories, the paleontological records of radiolarian assemblages from Japan Sea ODP Sites 794, 795, and 797 suggest that radiolarians have been influenced by the changing oceanography of the Japan Sea. The isolation of this marginal basin is illustrated by the lack of stratigraphic indicator species found in the sediments of the North Pacific (such as species found by Sakai, 1980). The sporadic occurrence (with 
common abundances) of radiolarians such as Lamprocyrtis heteroporos, and Eucyrtidium matuyamai and the occurrence of several stratigraphic indicators found outside the Japan Sea suggest that although there was communication between the Japan Sea and the Pacific throughout the Miocene and Pliocene, the water mass present in the Japan Sea must have had properties unique to this marginal basin and mixing with the Pacific was limited.

Tectonic changes as well as climatic changes of the Japan Sea have played a very important role in shaping the late Pliocene and Pleistocene Leg 127 radiolarian assemblages. Subtropical radiolarians in Japan Sea sediments record the influence of the sill at the Tsushima Strait, and the opal dissolution transition zone reflects communication with the Pacific at the Tsugaru Strait (White and Alexandrovich, this volume). The fact that many of the Miocene and Pliocene deposits studied on Japan contain radiolarian species common in the North Pacific (e.g., Nakaseko and Sugano, 1973), whereas most sediments of this age recovered during Leg 127 are lacking these same species, implies that the intra-arc deep-sea basins (Iijima and Tada, 1990) where these sediments were deposited had a more extensive connection with the waters of the North Pacific than did the major basins within the Japan Sea and that the presence of the islands of Japan formed a significant barrier between the Pacific and the Japan Sea.

\section{SUMMARY}

Radiolarians from the Japan Sea Leg 127 sediments are diverse and many interesting morphotypes are found. There are 25 relatively reliable biostratigraphic events found in these sediments, and because there are many forms of radiolarians present in these samples that have not been identified, more events are likely to be found. Depthdepth plots of the radiolarian datum levels from Sites 794, 795, and 797 show that additional stratigraphic evaluation is needed to refine the stratigraphic resolution of the radiolarian datum levels and correlations between sites.

Sediments of the Leg 127 sites provide excellent material for study of the controls of radiolarian preservation because several states of preservation are observable in the assemblages. The differing states of radiolarian preservation in the Japan Sea sediments record several different processes. Radiolarian assemblages from sediments in the Japan Sea with high indices of refraction suggest that dissolution probably occurred after deposition, either on the seafloor or within the sediment column, whereas concentrations of dissolution-resistant forms and/or radiolarian fragments suggest that dissolution probably occurred during deposition.

Compositional and preservational changes of radiolarians found in the Leg 127 sediments resulted from the unique oceanographic conditions in the Japan Sea and can be used to trace fluctuations of the paleo-Tsushima current, and the dissolution of radiolarian tests that may be related to changes in productivity, dissolved silicon concentration, and tectonics. Faunal changes observed in the Site 797 and 794 sediments indicate that the Japan Sea has been influenced by the Tsushima current as early as the late Pliocene or early Pleistocene $(\sim 1.8 \mathrm{Ma})$. The absence of biostratigraphic events recorded in the Pacific suggests that sill depths played an important role in determining the species composition of the radiolarian assemblages.

Because the evolutionary taxonomy of radiolarians found in the Japan Sea sediments is rather poorly developed, the cores from the Leg 127 sites provide a wealth of material for further study. Qualitative observations of the Leg 127 radiolarian assemblages revealed that there are many interesting morphological changes observable in radiolarian lineages, but evolutionary relationships between morphotypes are complex. There are a number of very unusual, as well as less distinctive, forms of radiolarians that have unknown species affiliations present in the samples, and large fluctuations in the ratio between spumellarian and nassellarian radiolarians may be an important paleontological record. Future studies that quantify the radiolar- ian paleontology of sediments obtained during Leg 127 are sure to reveal many interesting facts.

\section{ACKNOWLEDGMENTS}

Funding for this research was provided by the JOI U.S. Science Support Program. The laboratory assistance of Elaine Stock, and manuscript reviews by Joe Morley, Dick Casey, and Jim Ling are greatly appreciated.

\section{REFERENCES}

Bailey, J. W., 1856. Notice of microscopic forms found in the Sea of Kamtschatka - with a plate. Am. J. Sci., 22:1-6.

Benson, R. N., 1966. Recent Radiolaria from the Gulf of California [Ph.D. dissert.]. Minnesota Univ.

Campbell, A. S., and Clark, B. L., 1944. Miocene radiolarian faunas from Southern California. Spec. Pap.-Geol. Soc. Am., 51:1-76.

Dreyer, F., 1889. Morphologische Radiolarien studein, I, Die Pylombildungen in vergleitschend-anatomisher und entwicklungsgeschichlicher Bezeihung bei Radiolarien und Bei Protisten Uberhaupt, nebst System und Beschreibung neuer und des bis jetzt bekannten pylomatischen Spumellarien. Jena. Z. Naturwiss., 23:1-138.

Ehrenberg, C. G., 1862. Über den Tiefgrund-Verhaltnisse des Oceans am Eingange der Davisstrasse und bei Island. K. Preuss. Akad. Wiss. Berlin Monatsber., 1861:275-315.

Goll, R. M., 1968. Classification and phylogeny of Cenozoic Trissocyclidae (Radiolaria) in the Pacific and Caribbean basins. Part I. J. Paleontol., 42:1409-1432.

Haeckel, E., 1887. Report on the Radiolaria collected by H.M.S. Challenger during the years 1873-1876. Rep. Sci. Results Voy. Challenger, Zool., 18:1-1803.

Hays, J. D., 1965. Radiolaria and late Tertiary and Quaternary history of Antarctic Seas. In Llano, G. A. (Ed.), Biology of the Antarctic Seas II. Am. Geophys. Union, Antarct. Res. Ser., 5:125-184.

, 1970. Stratigraphy and evolutionary trends of Radiolaria in North Pacific deep-sea sediments. In Hays, J. D. (Ed.), Geological Investigations of the North Pacific. Mem.-Geol. Soc. Am., 126:185-218.

Hays, J. D., Imbrie, J., and Shackleton, N. J., 1976. Variations in the Earth's orbit: pacemaker of the ice ages. Science, 194:1121-1132.

Holdsworth, B. K., and Harker, B. M., 1975. Possible indicators of degree of Radiolaria dissolution in calcareous sediments of the Ontong-Java Plateau. In Andrews, J. E., Packham, G., et al., Init. Repts. DSDP, 30: Washington (U.S. Govt. Printing Office), 489-497.

Huang, T., 1967. A new Radiolarian from the Somachi Formation, Kikai-Jima, Kagoshima Prefecture, Japan. Trans. Proc. Paleontol. Soc. Jpn., 68:177-184.

Iijima, A., and Tada, R., 1990. Evolution of Tertiary sedimentary basins of Japan in reference to opening of the Japan Sea. J. Fac. Sci., Univ. Tokyo, 22:121-171.

Karig, D. E., Ingle, J. C., Jr., et al., 1975. Init. Repts. DSDP, 31: Washington (U.S. Govt. Printing Office).

Kling, S. A., 1973. Radiolaria from the eastern North Pacific, Deep Sea Drilling Project, Leg 18. In Kulm, L. D., von Huene, R., et al., Init. Repts. $D S D P, 18$ : Washington (U.S. Govt. Printing Office), 617-671.

Ling, H. Y., 1975. Radiolaria: Leg 31 of the Deep Sea Drilling Project. In Karig, D. E., Ingle, J. C., Jr., et al., Init. Repts. DSDP, 31: Washington (U.S. Govt. Printing Office), 703-760.

Lombari, G., and Lazarus, D. B., 1988. Neogene Cycladophorid radiolarians from North Atlantic, Antarctic, and North Pacific deep-sea sediments. Micropaleontology, 34:97-135.

Matoba, Y., 1984. Paleoenvironment of the Sea of Japan. In Oertli, H. J. (Ed.), Benthos '83: Proc. 2nd Int. Conf. Benthic Foraminifera, 409-414.

Moore, T. C., Jr., 1973. Method of randomly distributing grains for microscopic examination. J. Sediment. Petrol., 43:904-906.

Morley, J. J., and Hays, J. D., 1979. Cycladophora davisiana: a stratigraphic tool for Pleistocene North Atlantic and interhemispheric correlation. Earth Planet. Sci. Lett., 44:383-389.

, 1983. Oceanographic conditions associated with high abundances of the radiolarian Cycladophora davisiana. Earth Planet. Sci. Lett., 66:63-72.

Morley, J. J., Heusser, L. E., and Sarro, T., 1986. Latest Pleistocene and Holocene Palaeoenvironment of Japan and its marginal sea. Palaeogeogr., Palaeoclimatol., Palaeoecol., 53:349-358. 
Müller, J., 1858. Über die Thalassicollen, Polycystinen und Acanthometren des Mittelmeeres. K. Preuss. Akad. Wiss. Berlin Abh., 1-62.

Nakaseko, K., 1963. Neogene Cyrtoidea (Radiolaria) from the Isozaki Formation in Ibaraki Prefecture, Japan. Sci. Rep. Osaka Univ., 12:165-198. 1969. Neogene Radiolaria in Japan. Proc. Ist Inter. Conf. Planktonic Microfossils, 2:468-474.

1972. On some species of the genus Thecosphaera from the Neogene formations, Japan. Sci. Rep., Coll. Gen. Educ., Osaka Univ., 20:59-70.

Nakaseko, K., Iwamoto, H., and Takahashi, K., 1965. Radiolarian stratigraphy in the oil and gas bearing Tertiary and Upper Cretaceous formations, Japan. Contrib. from Govt. of Japan to Econ. Comm. for Asia and the Far East Third Pet. Symp., 1-13.

Nakaseko, K., and Sugano, K., 1973. Neogene radiolarian zonation in Japan. Jpn. Mem., 8:23-41.

Nigrini, C. A., 1977. Tropical Cenozoic Artostrobiidae (Radiolaria). Micropaleontology, 23:241-269.

Oba, T., Kato, M., Kitazato, H., Koizumi, I., Omura, A., Sakai, T., and Takayama, T., 1991. Paleoenvironmental changes in the Japan Sea during the last 85,000 years. Paleoceanography, 6:499-518.

Popofsky, A., 1908. Die Radiolarien der Antarktis (mit Ausnahme der Tripleen). Dtsch. Sudpolar-Exped. 1901-1903, 10 (Zool. Vol. 2):183-305.

Reynolds, R. A., 1980. Radiolarians from the western North Pacific, Leg 57 Deep Sea Drilling Project. In von Huene, R., Nasu, N., et al., Init. Repts. DSDP, 56, 57 (Pt. 2): Washington (U.S. Govt. Printing Office), 735-769.

Riedel, W. R., 1953. Mesozoic and late Tertiary Radiolaria of Rotti. J. Paleontol., 27:805-831.

, 1958. Radiolaria in Antarctic sediments. Rep. B.A.N.Z. Antarct. Res. Exped., Ser. B, 6:217-255.

Riedel, W. R., and Foreman, H. P., 1961. Type specimens of North American Paleozoic Radiolaria. J. Paleontol., 35:628-632.

Robertson, J. H., 1975. Glacial to interglacial oceanographic changes in the northwest Pacific, including a continuous record of the last 400,000 years [Ph.D. dissert.]. Columbia Univ., New York.

Sakai, T., 1980. Radiolarians from Sites 434, 435, and 436, Northwest Pacific, Leg 56, Deep Sea Drilling Project. In von Huene, R., Nasu, N., et al., Init. Repts. DSDP, 56, 57 (Pt. 2): Washington (U.S. Govt. Printing Office), 695-733.

Sanfilippo, A., and Riedel, W. R., 1970. Post-Eocene "closed" theoperid radiolarians. Micropaleontology, 16:446-462.

, 1980. A revised generic and suprageneric classification of the Artiscins (Radiolaria). J. Paleontol., 54:1008-1011.

Tamaki, K., Pisciotto, K., Allan, J., et al., 1990. Proc. ODP, Init. Repts., 127: College Station, TX (Ocean Drilling Program).

\section{Date of initial receipt: 9 May 1991 \\ Date of acceptance: 27 September 1991 \\ Ms 127/128B-134}

\section{APPENDIX}

\section{Systematic Species List}

Radiolarians species discussed and figured in the plates of this report are listed below in alphabetical order. References to original and nominal descriptions and some taxonomic notes are provided.

\section{Anthocorys akitaensis Nakaseko, 1969}

(PI. 5, Figs. 1, 2, and 3)

Botryostrobus bramlettei (Campbell and Clark, 1944) Nigrini, 1977

Clathrocyclas cabrilloensis Campbell and Clark, 1944

(Pl. 5, Figs. 7, 8, 9, and 10)

Cycladophora robusta Lombari and Lazarus, 1988

(PI. 5, Figs. 11 and 12)

Cyrtocapsella tetrapera Haeckel, 1887

(PI. 2, Figs. 4 and 5)
Didymocyrtis tetrathalamus (Haeckel, 1887) Sanfilippo and Riedel, 1980 (Pl. 4, Fig. 3)

Euchitonia species (Pl. 4, Fig. 7)

Remarks. Specimens identified as belonging to the genus Euchitonia probably belong to the species Euchitonia elegans, Euchitonia furcata, or Euchitonia triangulum.

$$
\text { Eucyrtidium matuyami Hays, } 1970
$$

Lamprocyrtis heteroporos (Hays, 1965) Kling, 1973

Larnacantha polyacantha Campbell and Clark, 1944 (Pl. 4, Fig. 5)

Remarks. This species is equivalent to Phorticium pylonium Haeckel, used in faunal analyses of Robertson (1975), and Morley et al. (1986).

Lipmanella sp. aff. Theocorys redondoensis Reynolds, 1980

$$
\text { (Pl. 3, Fig. 4) }
$$

Lithatractus tochigiensis Nakaseko, 1969

(Pl. 3, Fig. 10)

Lychnocanium nipponicum Nakaseko, 1963

(Pl. 3, Fig. 8)

Lychnocanium sp. cf L. grande Campbell and Clark, 1944

$$
\text { (Pl. 3, Fig. 1) }
$$

Peripyramis species

(Pl. 2, Fig. 3, and Pl. 4, Fig. 5)

Remarks. It is hard to identify radiolarians belonging to this genus to the species level, due to the effects of dissolution. Species that may be represented in this category include Peripyramis circumtexta Haeckel, 1887 and Plectopyramis pacifica Nakaseko, 1963.

Sethocyrtis japonica Nakaseko, 1963

(Pl. 3, Fig. 9)

Remarks. This species actually belongs to the genus Theocorys (Reynolds, 1980), however the genus name Sethocyrtis has been retained in order to avoid confusion in discussions between this species and the spumellarian $T$. (Thecosphaera) japonica.

Sphaeropyle langii Dreyer, 1889

(Pl. 1, Fig. 7, and Pl. 3, Fig. 3)

Sphaeropyle robusta Kling, 1973

(Pl. 3, Fig. 7)

Spongaster tetras Ehrenberg 1862

Spongotrochus glacialis Popofsky, 1908

(PI. 4, Fig. 4)

Stichocorys delmontensis (Campbell and Clark, 1944) Sanfilippo and Riedel, 1970

Stichocorys peregrina (Riedel, 1953) Sanfilippo and Riedel, 1970

Stichocorys wolfii Haeckel, 1887

Stylacontarium acquilonius (Hays, 1970) Kling, 1973

$$
\text { (Pl. 3, Fig. 2) }
$$

Stylacontarium sp. cf. S. acquilonarium (Kling, 1973) Reynolds, 1980

Stylatractus universus Hays, 1970 
Stylochlamydium venustum (Bailey, 1856) Haeckel, 1887 (Pl. 4, Fig. 6)

Tetrapyle octacantha Müller, 1858

(PI. 4, Figs. 1 and 2)

Theocorys redondoensis (Campbell and Clark, 1944) Kling, 1973 (Pl. 3, Fig. 5)

Thecosphaera akitaensis Nakaseko, 1972
Thecosphaera japonica Nakaseko, 1972 (Pl. 3, Fig. 11)

Theocalptra davisiana (Ehrenberg, 1862) Riedel, 1958 (Pl. 1, Fig. 6, and Pl. 4, Fig. 8)

Tholospyris anthopora (Haeckel, 1887) Goll, 1968

Tholospyris mammillaris (Haeckel, 1887) Goll, 1968 (PI. 3, Fig. 6) 


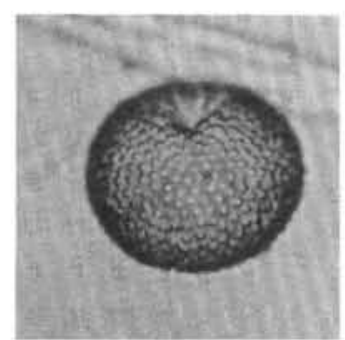

1

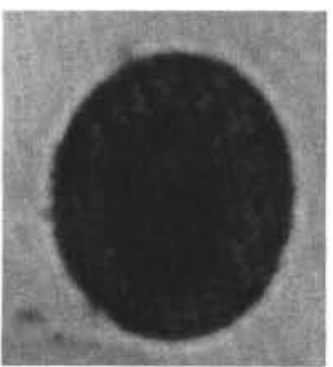

3

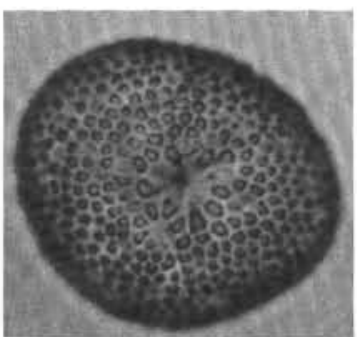

2

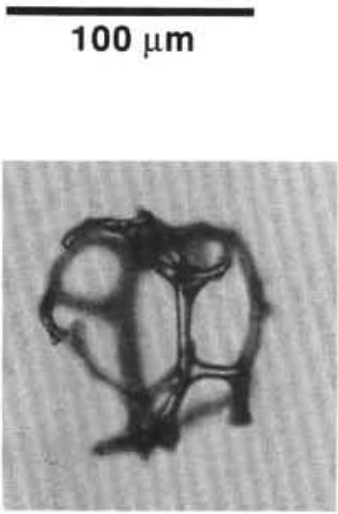

4

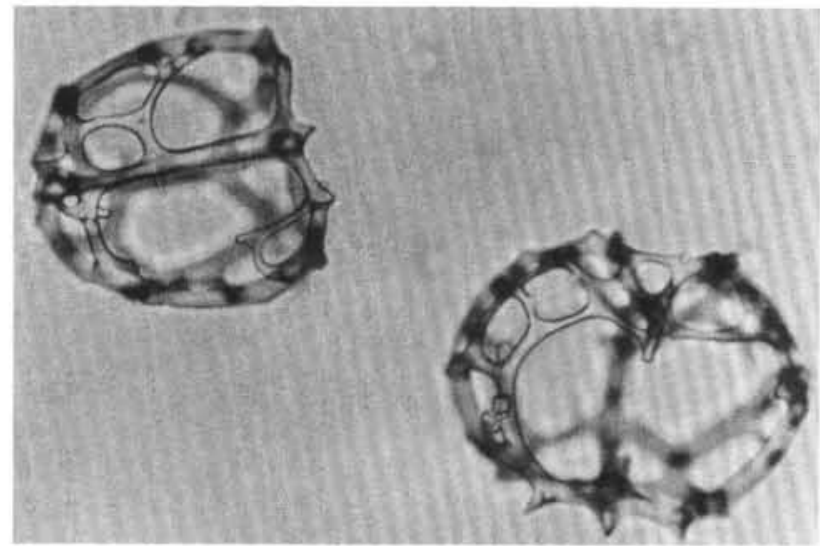

5

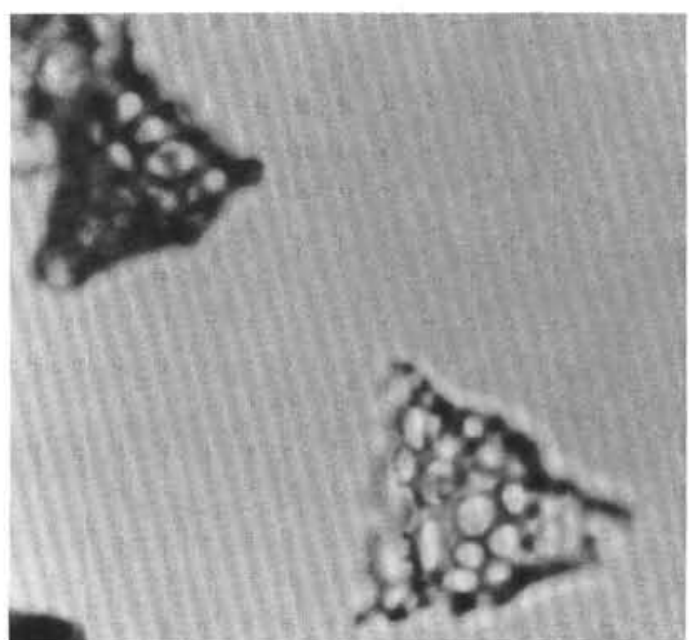

6

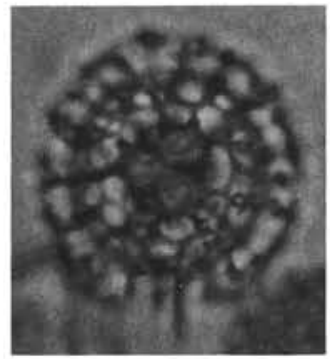

7

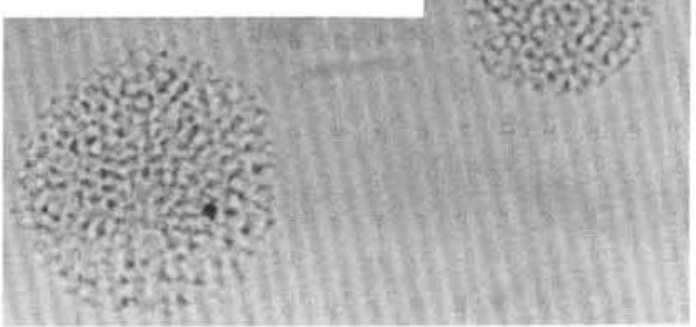

8

Photographs presented in the following plates were taken using a 35-mm camera mounted on a beam splitter on a Wild M20 microscope. All figures are at the same scale $(2.5 \mathrm{~cm}=100 \mu \mathrm{m})$. EF refers to the England Finder location (Riedel and Foreman, 1961) of the specimen on the sample slide. Samples are kept at the Department of Geology, Florida Atlantic University.

Plate 1. Sterraster sponge spicules and radiolarian indicators of dissolution. 1-3. Sterraster sponge spicules: (1) Sample 127-794A-1H-3, 60-62 cm, EF T51/1; (2) Sample 127-797B-26X-5, 108-110 cm, EF L47/4; (3) Sample 127-794A-1H-5, 55-57 cm, EF F40. 4, 5. Trissocyclid radiolarians: (4) Highly dissolved trissocyclid radiolarian representing the only radiolarian recovered from Sample 127-794A-1H-3, 60-62 cm, EF M43; (5) 127-797B-10H-4, 118-120 cm, EF O39. 6. Theocalyptra davisiana. These two specimen show two states of index of refraction of the same species in Sample 127-795A-12H-1, 70-72 cm, EF Q40/3. Top left has low index of refraction and bottom right has high index of refraction. 7. Sphaeropyle langii. Partially dissolved or juvenile specimen missing cortical shell from Sample 127-795A-12H-5, 70-72 cm, EF L35/3. 8. Spongodiscid radiolarians displaying high index of refraction from Sample 127-797B-4H-1, 118-120 cm, EF W44/3. 


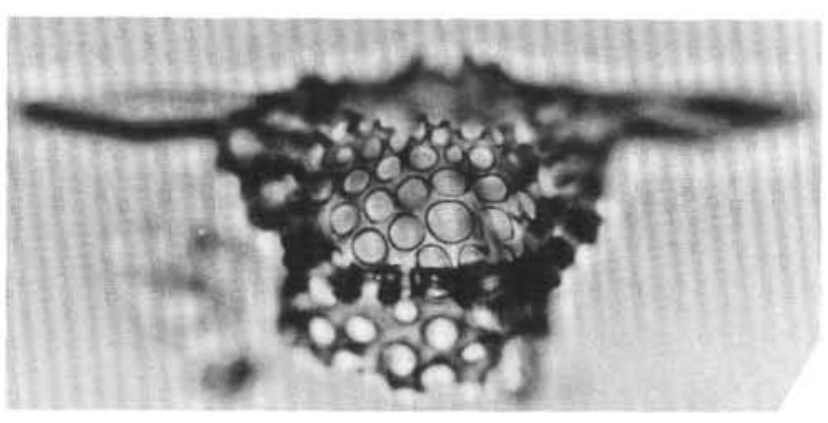

1

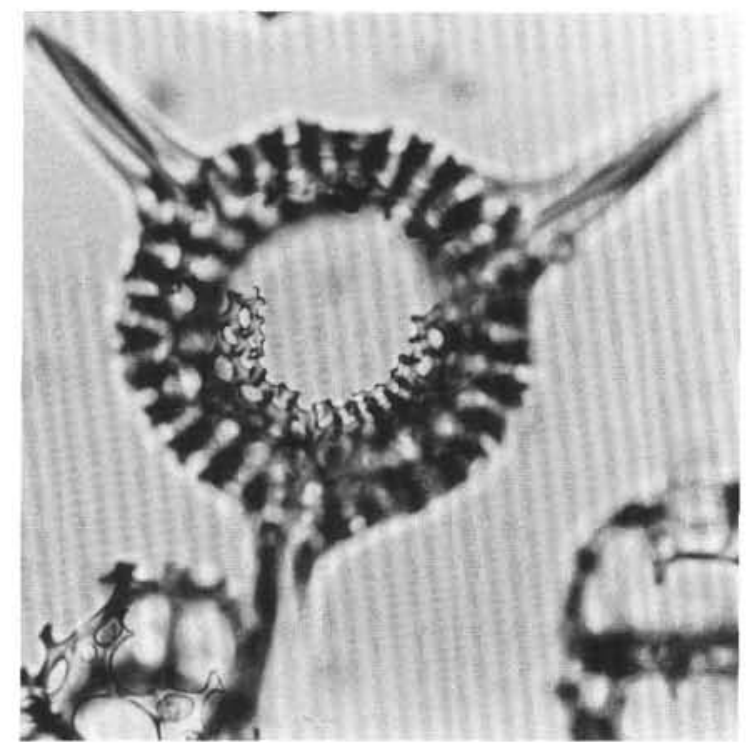

2

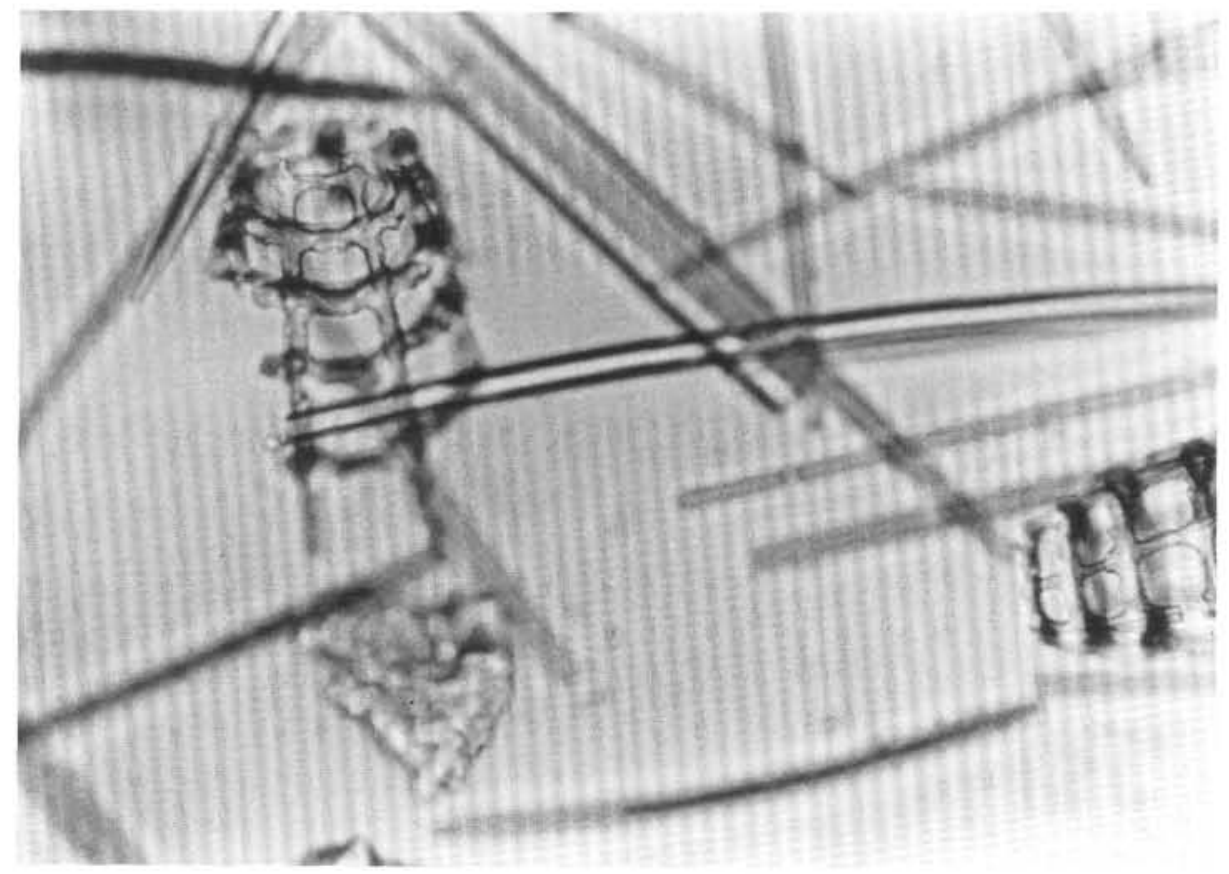

3

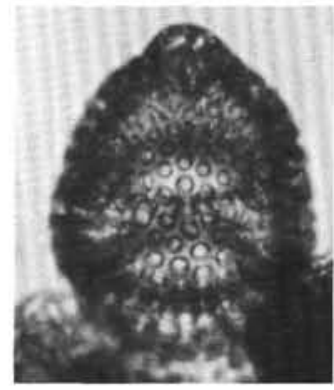

4

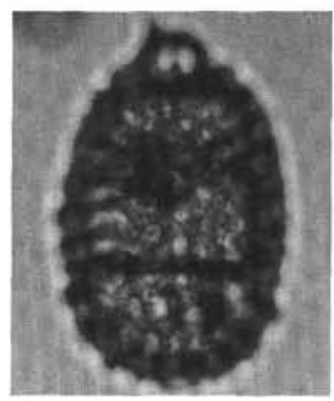

5

Plate 2. Radiolarian indicators of dissolution and reworking. 1, 2. Two views of an unidentified nassellarian radiolarian, present in high abundance, but found only as broken specimens indicating disturbance and reworking. Both figures are from Sample 127-794A-4H-6, 60-62 cm: (1) EF U46/2; (2) EF M37/3. The presence of high numbers of trissocyclid radiolarians (Fig. 2) in this sample is another indicator that this sample has been subjected to high levels of dissolution. 3. Highly dissolved specimen of the species Peripyramis circumtexta or Circumtexta pacifica, and sponge spicules in Sample 127-795A-12H-2, 70-72 cm, EF V25/4. 4, 5. Cyrtocapsella tetrapera: (4) found as an indicator of reworking in Pleistocene Sample 127-797B-7H-2, 118-129 cm, EF P48/1; and (5) within its actual stratigraphic range in Sample 127-794B-5R-CC, EF V13. 

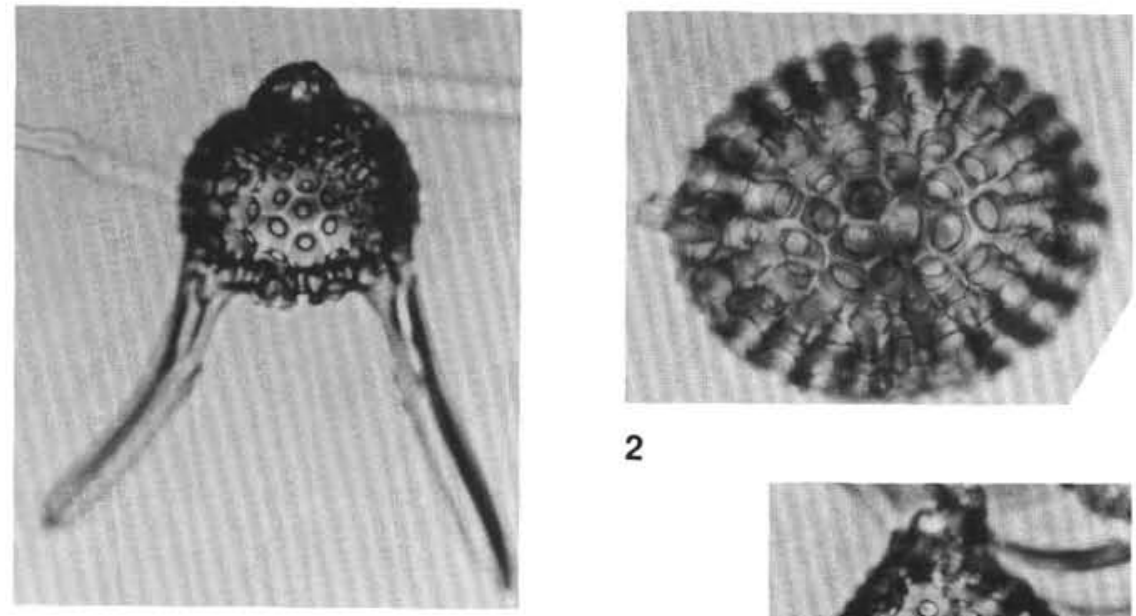

2

1

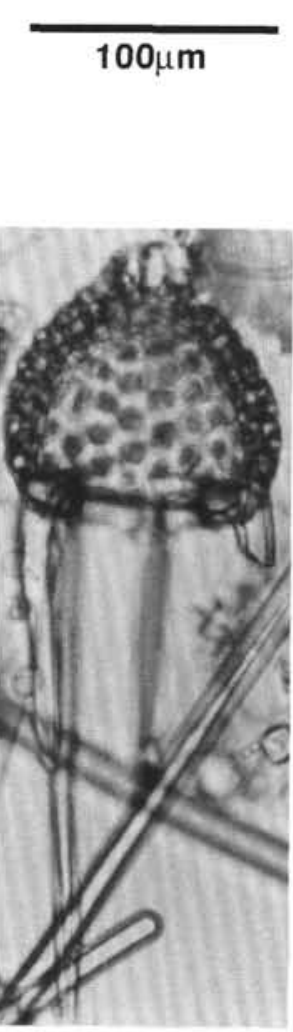

8

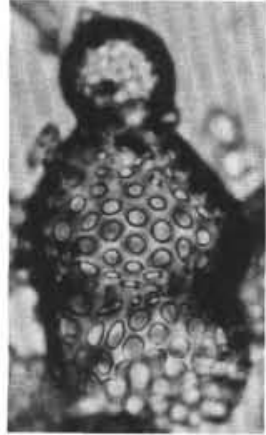

5

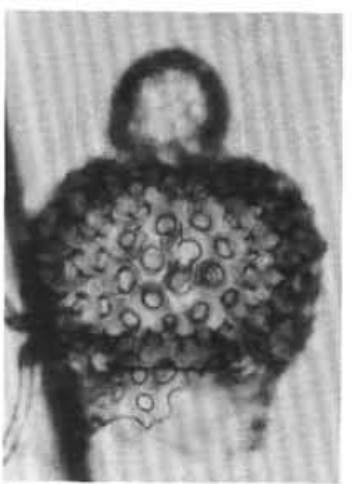

9

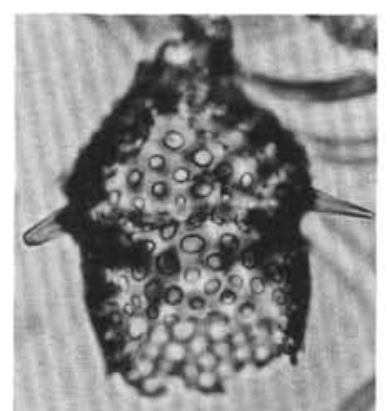

4

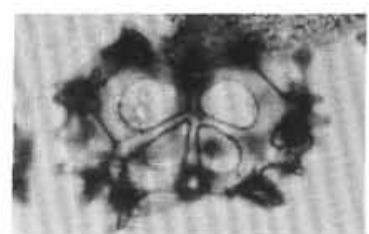

6

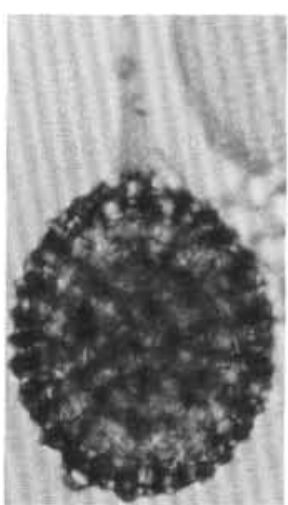

10

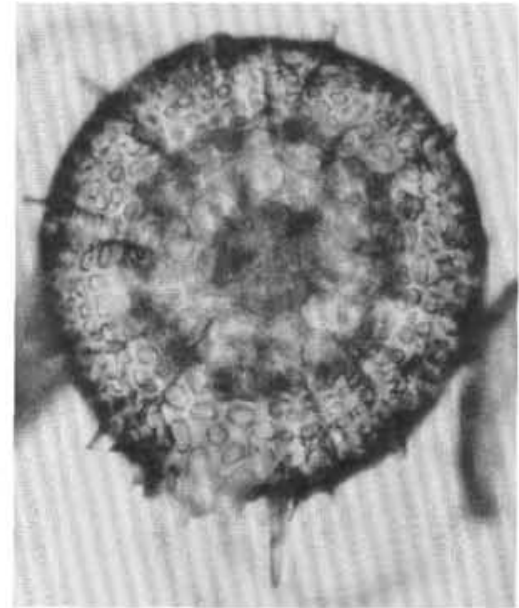

3

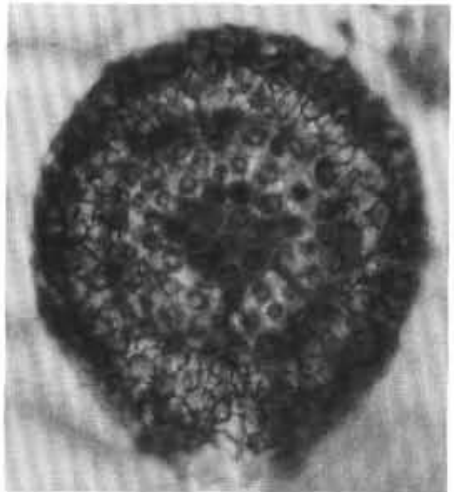

7

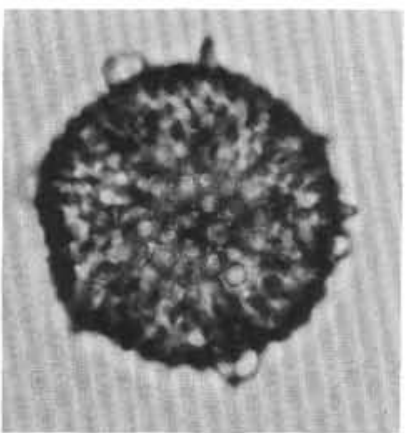

11

Plate 3. Japan Sea radiolarian stratigraphic indicators. 1. Lychnocanium sp. cf L grande. Sample 127-797B-2H-5, 118-120 cm, EF M52. 2. Stylacontarium acquilonius. Sample 127-794A-7H-6, 60-62 cm, EF Q31. 3. Sphaeropyle langii. Sample 127-794A-11H-3, 60-62 cm, EF H2/3. 4. Lipmanella sp. aff. Theocorys redondoensis. Sample 127-794A-19X-2, 60-62 cm, EF R40/4. 5. Theocorys redondoensis. Sample 127-794A-19X-1, 60-62 cm, EF Y43. 6. Tholospyris mammilaris. Sample 127-794A-29X-1,60-62 cm, EF X29/3. 7. Sphaeropyle robusta. Sample 127-797B-23X-5,108-110 cm, EF S4. 8. Lychnocanium nipponicum. Sample 127-797A-26X-5, 108-110 cm, EF G33/3. 9. Sethocyrtis japonica. Sample 127-794A-28X-2,60-62 cm, EFZ47/4. 10. Lithatractus tochigiensis. Sample 127-794A$29 \mathrm{X}-1,60-62 \mathrm{~cm}, \mathrm{EF}$ V35/3. 11. The cosphaera japonica. Sample 127-795A-12H-1, 70-72 cm, EF R49. 


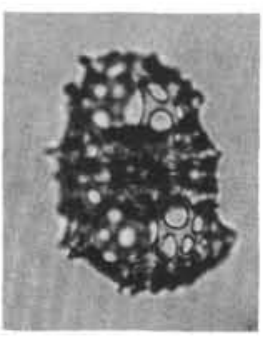

1

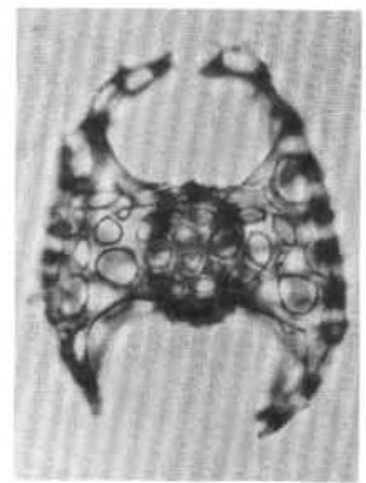

2

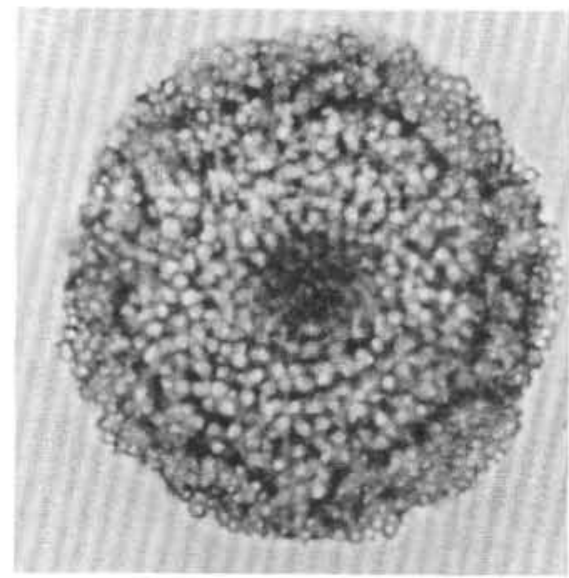

6

3

4
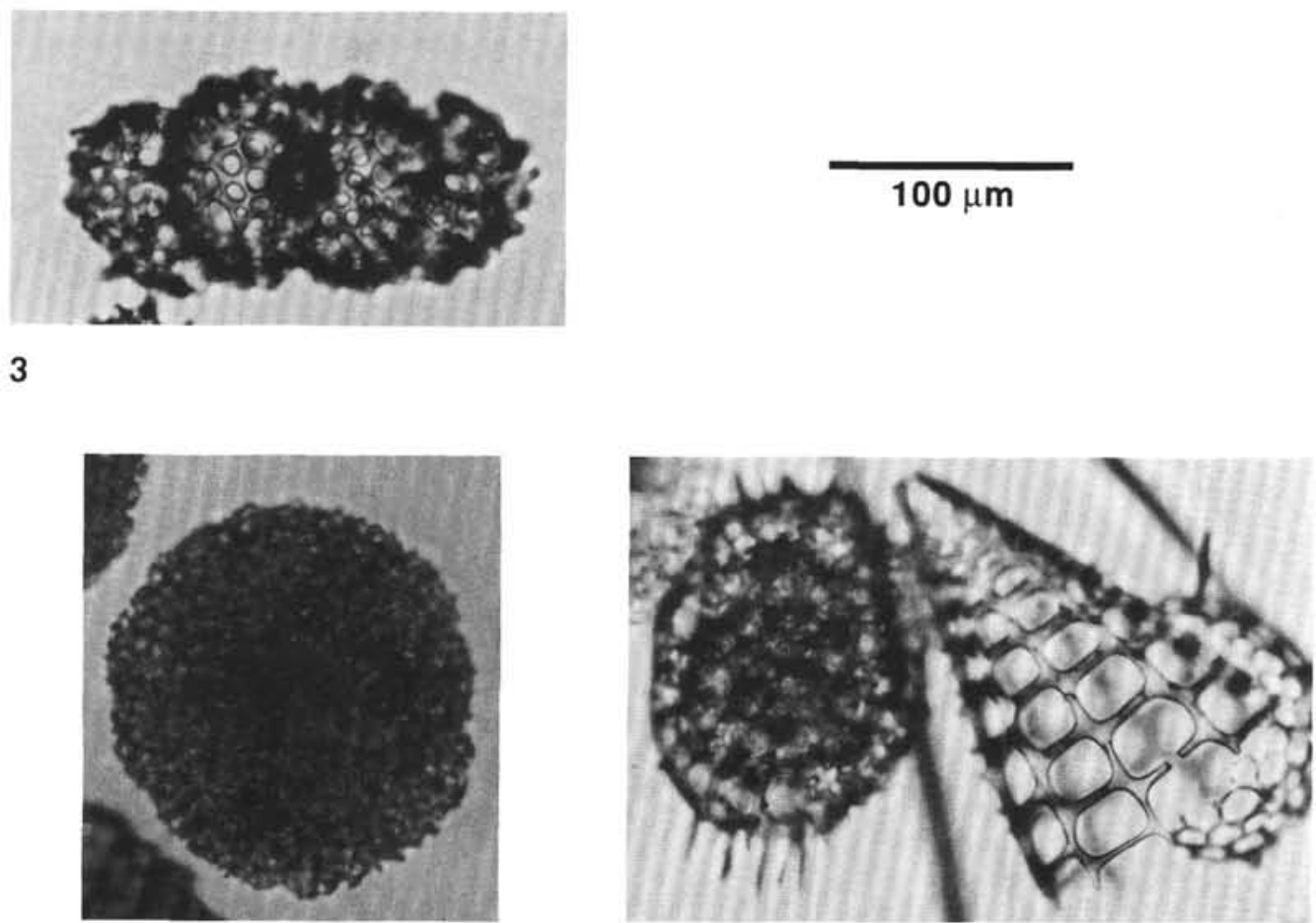

5
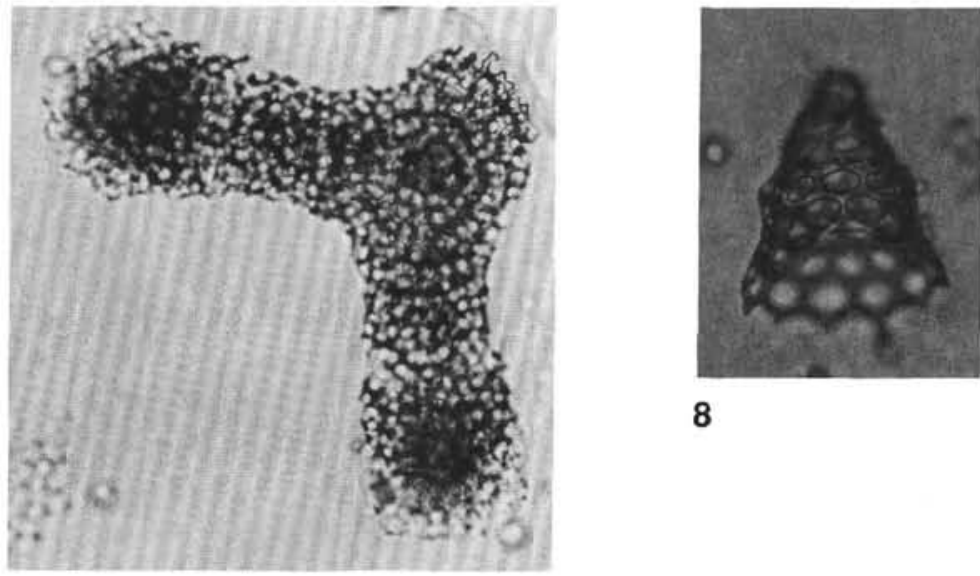

8

Plate 4. Japan Sea radiolarian paleoceanographic indicators. 1, 2. Tetrapyle octacantha: (1) Sample 127-794A-6H-2, 59-61 cm, EF X47/2; (2) Sample 127-797B-7H-3, 117-119 cm, EF Y43/4. 3. Didymocyrtis tetrathalamus. Sample 127-797B-8H-4, 118-120 cm, EF T45. 4. Spongotrochus glacialis. Sample 127-795A-19H-4, 73-75 cm, EF H41/3. 5. Larnacantha polyacantha on left and Peripyramis species on right. Sample 127-795A-15H-1, 73-75 cm, EF C33/2. 6. Stylochlamydium venustum. Sample 127-797B-8H-1, 118-120 cm, EF R20/1. 7. Euchitonia species. Sample 127-794A-3H-2, 60-62 cm, EF X16. 8. Theocalyptra davisiana. Sample 127-794A-6H-1, 59-61 cm, EF W11. 


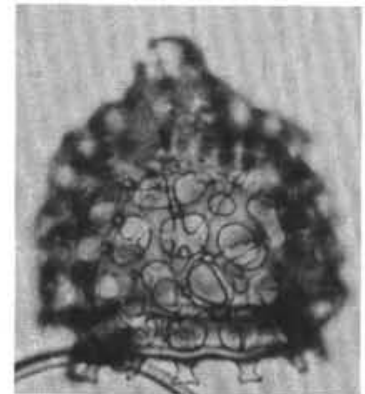

1

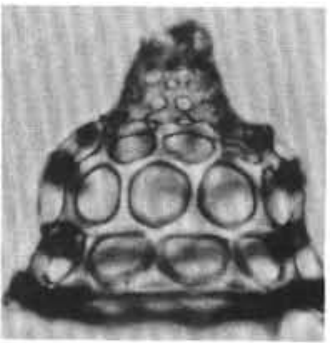

4

$100 \mu \mathrm{m}$

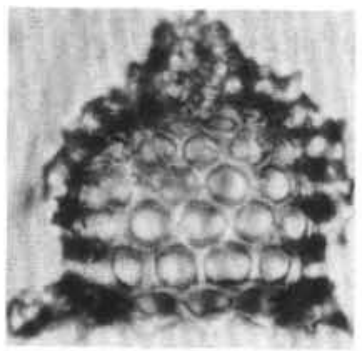

2
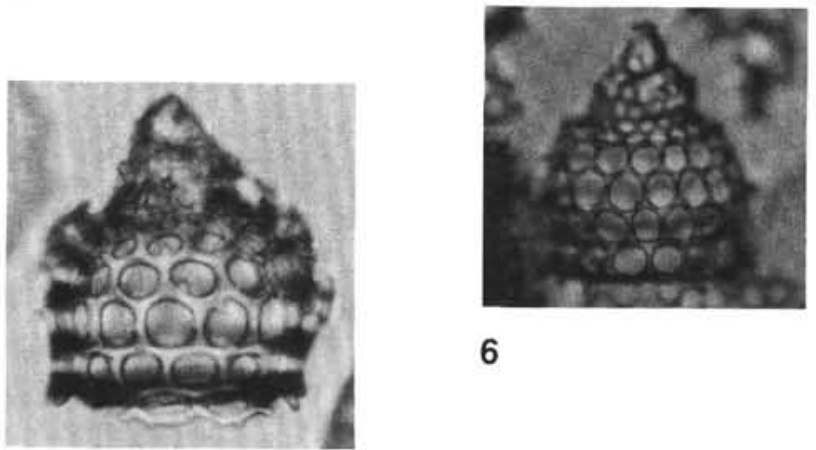

6

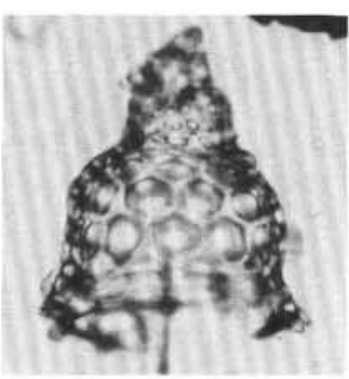

7

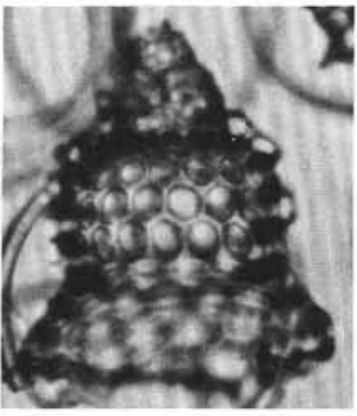

9

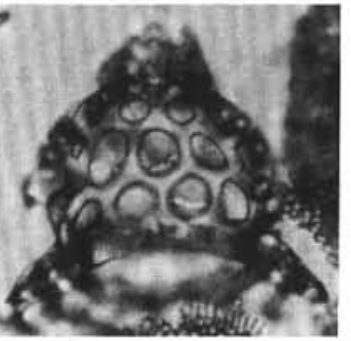

11

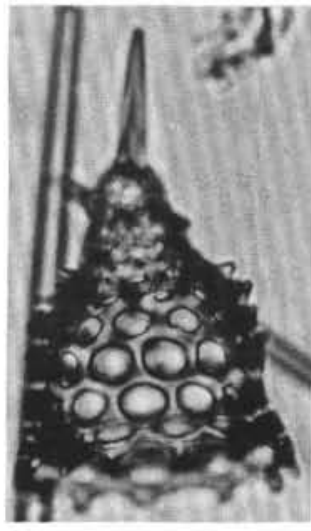

8

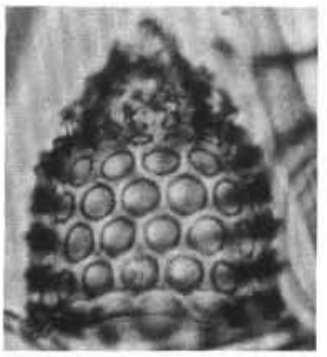

10

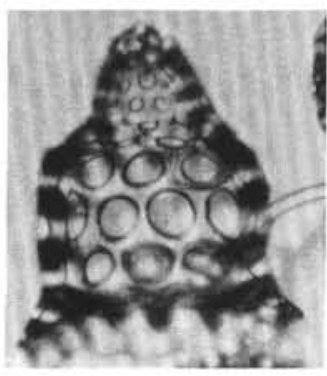

12

Plate 5. Synchronopticon of Cycladophorid radiolarians showing some of the variability of these forms in Japan Sea sediments. Horizontal level of the top of each photo represents relative age of radiolarian (youngest at top, oldest at bottom. Ages listed with samples are calculated using the age model listed in Tamaki et al. (1990) and summarized in White and Alexandrovich (this volume). 1-3. Anthocorys akitaensis: (1) age $=0.29 \mathrm{Ma}$, Sample 127-797B-2H-5, 118-120 cm, EF N21; (2) age = 3.3 Ma, Sample 127-797B-17H-7, 118-120 cm, EF J25/4; (3) age = 5.3 Ma, Sample 127-797B-26X-5, 108-110 cm, EF L47/4. 4-6. Unidentified Cycladophorid radiolarians: (4) age = 1.4 Ma, Sample 127-794A-6H-2, 59-61 cm, EF H26; (5) age = 2.7 Ma, Sample 127-794A-9H-6, 59-61 cm, EF S13/4; (6) age = 5 Ma, Sample 127-794A-17H-6, 60-62 cm, EF T25/1. 7-10. Clathrocyclas cabrilloensis: (7) age $=1.2 \mathrm{Ma}$, Sample 127-794A-5H-5, 58-60 cm, EF Q26; (8) age =1.1 Ma, Sample 127-797B-6H-4, 110-112 cm, EFT15/4; (9) age $=2.9 \mathrm{Ma}$, Sample 127-794A-10H-6,60-62 cm, EF K13; (10) age =3.8Ma, Sample 127-797A-20X-4, 108-110 cm, EFQ26/4. 11,12. Two specimens of Cycladophora robusta from Sample 127-794A-28X-2, 60-62 cm, age = 7.2 Ma: (11) with lace, EF W47; (12) without lace, EF X34/2. 